Article

\title{
In Vitro Antimycobacterial Activity and Physicochemical Characterization of Diaryl Ether Triclosan Analogues as Potential InhA Reductase Inhibitors
}

\author{
Tarek S. Ibrahim ${ }^{1,2, *}{ }^{\mathbb{C}}$, Ehab S. Taher ${ }^{3}$, Ebtihal Samir ${ }^{4}$, Azizah M. Malebari ${ }^{1}$, \\ Ahdab N. Khayyat ${ }^{1}(\mathbb{D})$, Mamdouh F. A. Mohamed ${ }^{5}\left(\mathbb{D}\right.$, Riham M. Bokhtia ${ }^{2,6}{ }^{(D}$, \\ Mohammed A. AlAwadh ${ }^{1}$, Israa A. Seliem ${ }^{2,6}$, Hani Z. Asfour ${ }^{7}$, Nabil A. Alhakamy ${ }^{8}$ (D, \\ Siva S. Panda ${ }^{6, *(D)}$ and Amany M. M. AL-Mahmoudy ${ }^{2}$ \\ 1 Department of Pharmaceutical Chemistry, Faculty of Pharmacy, King Abdulaziz University, \\ Jeddah 21589, Saudi Arabia; amelibary@kau.edu.sa (A.M.M.); ankhayyat@kau.edu.sa (A.N.K.); \\ Malawadh@kau.edu.sa (M.A.A.) \\ 2 Department of Pharmaceutical Organic Chemistry, Faculty of Pharmacy, Zagazig University, \\ Zagazig 44519, Egypt; rbokhtia@augusta.edu (R.M.B.); isliem@augusta.edu (I.A.S.); \\ amanysinger77@gmail.com (A.M.M.A.-M.) \\ 3 Department of Pharmaceutical Organic Chemistry, Faculty of Pharmacy, Al-Azhar University, \\ Assiut 71524,Egypt; ehaborganic@yahoo.com \\ 4 Physical Chemistry, Department of Analytical Chemistry, Faculty of Pharmacy, Deraya University, \\ New Minia 61519, Egypt; Ebtihal_samir@yahoo.com \\ 5 Department of Pharmaceutical Chemistry, Faculty of Pharmacy, Sohag University, Sohag 82524, Egypt; \\ mamdouhfawzy3@yahoo.com \\ 6 Department of Chemistry \& Physics, Augusta University, Augusta, GA 30912, USA \\ 7 Department of Medical Microbiology and Parasitology, Faculty of Medicine, King Abdulaziz University, \\ Jeddah 21589, Saudi Arabia; hasfour@kau.edu.sa \\ 8 Department of Pharmaceutics, Faculty of Pharmacy, King Abdulaziz University, Jeddah 21589, Saudi Arabia; \\ nalhakamy@kau.edu.sa \\ * Correspondence: tmabrahem@kau.edu.sa (T.S.I.); sipanda@augusta.edu (S.S.P.)
}

Academic Editors: Rachid Skouta and Mai Antonello

Received: 28 January 2020; Accepted: 6 July 2020; Published: 8 July 2020

\begin{abstract}
Two sets of diphenyl ether derivatives incorporating five-membered 1,3,4-oxadiazoles, and their open-chain aryl hydrazone analogs were synthesized in good yields. Most of the synthesized compounds showed promising in vitro antimycobacterial activity against Mycobacterium tuberculosis H37Rv. Three diphenyl ether derivatives, namely hydrazide 3, oxadiazole 4 and naphthylarylidene $8 \mathrm{~g}$ exhibited pronounced activity with minimum inhibitory concentrations (MICs) of $0.61,0.86$ and $0.99 \mu \mathrm{g} / \mathrm{mL}$, respectively compared to triclosan $(10 \mu \mathrm{g} / \mathrm{mL})$ and isoniazid (INH) $(0.2 \mu \mathrm{g} / \mathrm{mL})$. Compounds 3, 4, and $8 \mathrm{~g}$ showed the InhA reductase enzyme inhibition with higher $\mathrm{IC}_{50}$ values $(3.28-4.23 \mu \mathrm{M})$ in comparison to triclosan $(1.10 \mu \mathrm{M})$. Correlation between calculated physicochemical parameters and biological activity has been discussed which justifies a strong correlation with respect to the inhibition of InhA reductase enzyme. Molecular modeling and drug-likeness studies showed good agreement with the obtained biological evaluation. The structural and experimental information concerning these three InhA inhibitors will likely contribute to the lead optimization of new antibiotics for M. tuberculosis.
\end{abstract}

Keywords: diphenyl ether; H37Rv; antitubercular agents; InhA inhibitors; molecular docking 


\section{Introduction}

Tuberculosis (TB), caused mainly by Mycobacterium tuberculosis (MTB), remains the world's deadliest infection, having recently surpassed HIV as the leading cause of infectious disease mortality. The risk of developing tuberculosis (TB) is estimated as 16-27 times greater in people living with HIV than among those without HIV infection [1]. About 4500 people lose their lives, and close to 30,000 people fall ill each day with this preventable and curable disease [2]. The treatment plan for MTB infection is strenuous, requires long-term treatment of combination chemotherapy of two or more drugs, like pyrazinamide, isoniazid (INH), rifampicin, streptomycin and/or ethambutol (Figure 1). This combination usually accompanied by poor patient compliance, which leads to emerging of multi-drug resistant (MDR-TB) strains [3].

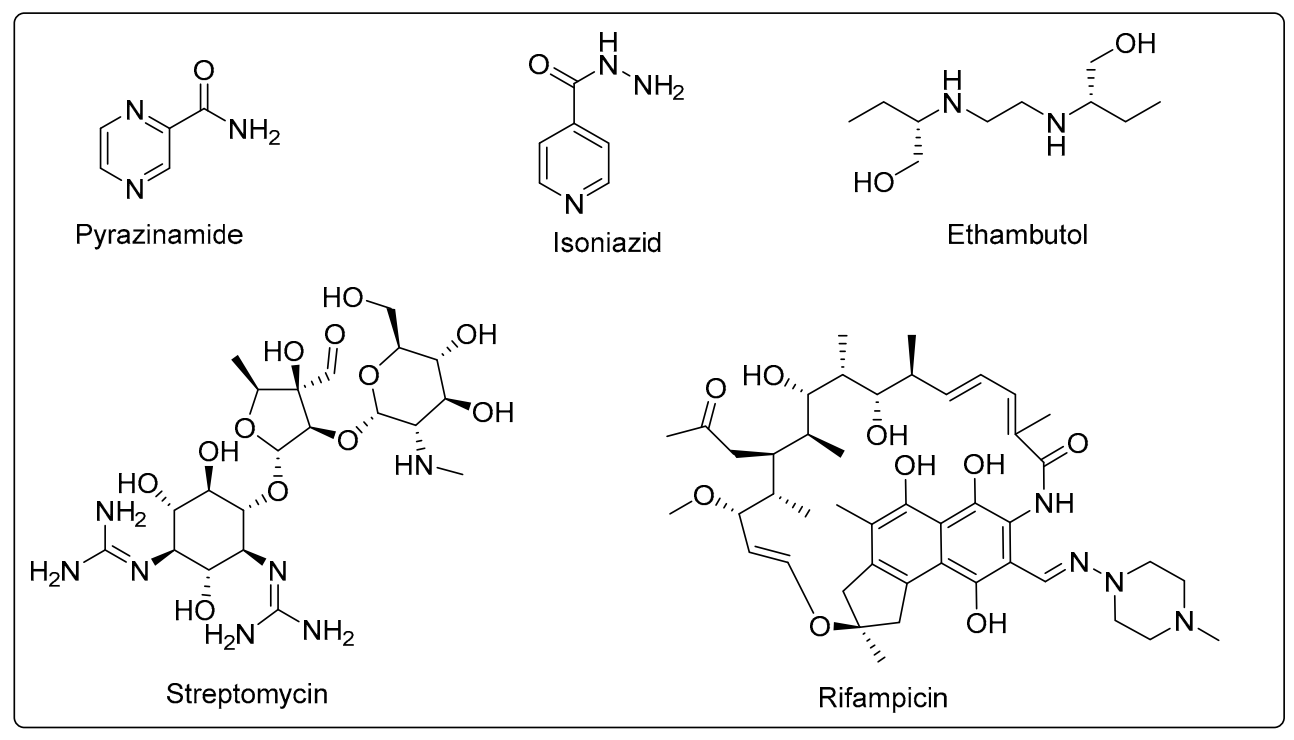

Figure 1. Commercially available anti-tubercular drugs.

Although one possible long-term solution to this issue is a better vaccine, in the near future, the major reliance will be on chemotherapy requiring an urgent development of potential, non-toxic, and effective antitubercular agents against latent MTB. In the search for novel TB lead candidates, the enoyl-acyl carrier protein, InhA reductase has become a target of interest by pharmaceutical companies and academic institutions for the development of new anti-tubercular drugs. InhA is the key enzyme that catalyzes NADH-dependent reduction of 2-trans-enoyl-ACP (acyl carrier protein) to give up $\mathrm{NAD}^{+}$and reduced enoyl thioester-ACP substrate, which in turn, aids in the synthesis of mycolic acid. Mycolic acid is a unique signature fatty acid and considered as a core constituent of the mycobacterial cell wall [4]. InhA enzyme has been identified as the key target of the most currently existing anti-TB drugs isoniazid (INH), and ethionamide. Most cases of resistance to these drugs result from mutations in the mycobacterial activating enzyme (catalase-peroxidase KatG for isoniazid and EthA for ethionamide) [5-13]. Also, triclosan (Figure 2), the non-ionic lipophilic anti-TB agent, has been identified as a promising reversible inhibitor of InhA. This diphenyl ether molecule doesn't require any bioactivation and capable of directly inhibit InhA. This approach probably allows avoiding the dominant cause of the resistance to isoniazid consisting of KatG mutations. SAR studies of triclosan revealed that chlorine atoms in ring B are not significant in the activity while ring $B$ itself is essential moiety for inhibition of InhA (Figure 2); it makes several hydrophobic contacts with adenoyl acyl carrier protein reductase enzyme. The ether oxygen bond is also crucial for the activity due to two reasons. Initially, it is necessary for maximizing the interaction of triclosan with InhA and consequently better biological activity [14]. Secondly, being of critical in the formation of the stable ternary InhA-triclosan-NAD ${ }^{+}$complex. Replacement of this group by a sulfur atom 
abolishes the inhibitory activity $[13,15]$. Different literature studies have been carried out based on the above hypothesis. Yang and coworkers carried out several of Des-chloro-diphenyl ether derivatives such as A and B (Figure 2), in the absence of the phenolic group, to enhance pharmacokinetic and pharmacodynamic properties thereby improving the druggability of triclosan. These compounds showed good antitubercular potency against MTB strain H37Rv (Figure 2) [16].

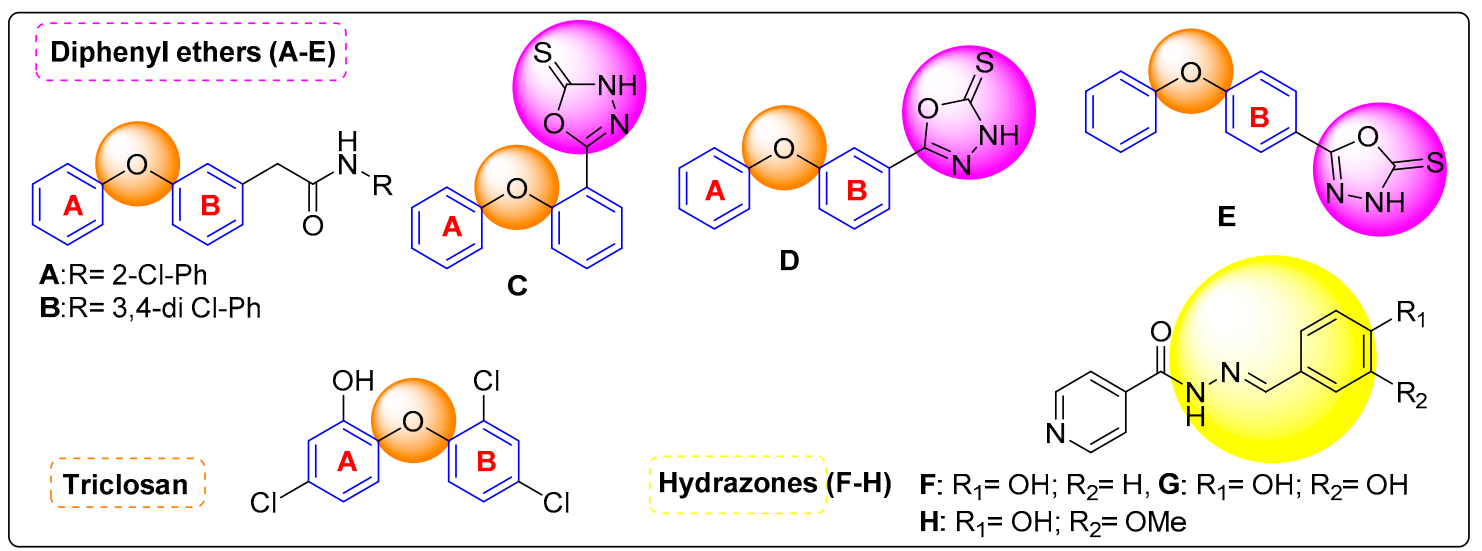

Figure 2. Structures of triclosan, diphenyl ether, and hydrazone derivatives.

On the other facet, oxadiazoles represent an important class of 5-membered heterocyclic systems that cover a broad range of biological activities such as antimycobacterial [17], pesticide [18], anti-inflammatory and analgesic [19], antimicrobial [20] and antitumor [21,22], In the search of more effective anti-tuberculosis, oxadiazole is one of the most versatile scaffolds against MTB strain. Interestingly, extensive chemical and structural investigations have been performed by Kini and his group through developing series of oxadiazole $o / m / p$-substituted diphenyl ether derivatives (C-E) which displayed inhibition against the growth of the H37Rv strain of mycobacterium (Figure 2) [9]. The hydrazide hydrazone skeleton is also a class of privileged structures with a broad range of biological activities such as antioxidant [23], anti-inflammatory [24], and antitubercular activities [25,26]. Recently, in a high throughput screening for inhibitors of $M$. tuberculosis $\mathrm{H} 37 \mathrm{Rv}$, several aryl hydrazine derivatives (F-H, Figure 2) [27] were found to possess potent activity in combination with the relatively low toxicity [27]. However, despite the promising in vitro activity of the currently existing diphenyl ethers have poor bioavailability [28] and limited aqueous solubility [28], which is likely one reason why they have limited in vivo efficacy [10].

In continuation of our previous work in parallel to the above findings and the observed relationship between lipophilicity and in vivo efficacy, especially as it pertains to antimicrobial agents [5-12], new diphenyl ether analogs have been designed as potential antitubercular inhibitors of InhA reductase enzyme. This approach developed by keeping the known essential functional groups for the activity. Modification has been made to the diphenyl ether ' $\mathrm{B}$ ' ring of diphenyl ether through binding the $\mathrm{B}$ ring with five-membered oxadiazole moieties either substituted or non-substituted ones; this might enhance bioactivity, pharmacokinetics or pharmacodynamics properties and could be a good lead for the generation of InhA reductase inhibitors. Furthermore, the substitution of the para position of diphenyl ether ' $\mathrm{B}$ ' ring with aryl hydrazine or arylidene moiety as an open-chain analog of oxadiazole may improve solubility and enhance the antimycobacterial activity. Prediction of correlation between antitubercular activity with both $\mathrm{Clog} \mathrm{P}$ and $\mathrm{IC}_{50}$ of InhA inhibition has been represented. Also, the correlation of the anti-TB activity with different thermodynamic, topological parameters was also considered. Molecular modeling studies were carried out to better understand the observed activity and determine the most important structural parameters participating in binding with InhA reductase that may affect the biological activity. 


\section{Results and Discussion}

\subsection{Chemistry}

The target derivatives were prepared through the conversion of the starting phenoxybenzoic acid 1 into the corresponding ester 2 using methanol and concentrated sulphuric acid under reflux conditions [28-30]. Hydrazinolysis of the ester 2 with hydrazine hydrate gives the hydrazide 3 (Scheme 1). Cyclocondensation of hydrazide 3 through its reaction with triethyl orthoformate under refluxing conditions led to the formation of the oxadiazole 4 (Scheme 2). The reaction of the hydrazide 3 with the appropriate aromatic carboxylic acid derivatives $5 \mathbf{a}-\mathbf{1}$ in the presence of phosphorus oxychloride afforded the corresponding 5 -aryl-substituted oxadiazoles $6 \mathbf{a}-\mathbf{1}$. The condensation reaction of hydrazide 3 with variant aromatic aldehydes $\mathbf{7} \mathbf{a}-\mathbf{k}$ resulted in the formation of anticipated arylidenes $\mathbf{8 a}-\mathbf{k}$ (Scheme 2). The prepared target compounds were characterized by spectral studies.

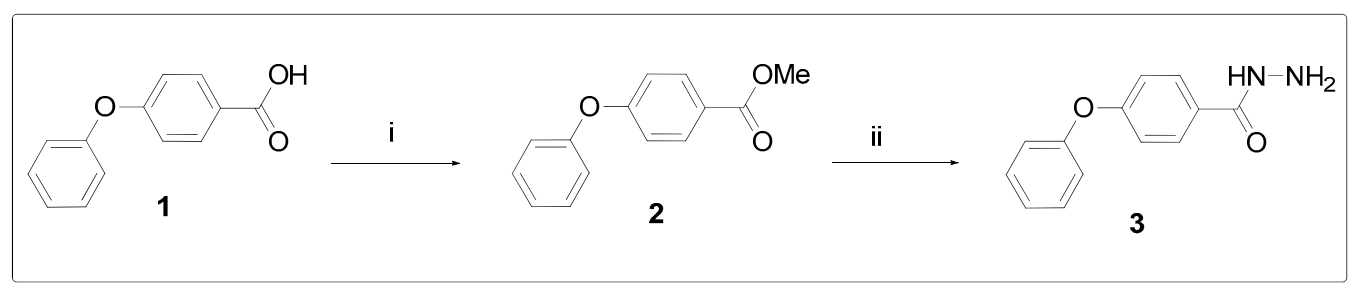

Scheme 1. Reagents and conditions: (i) Conc. $\mathrm{H}_{2} \mathrm{SO}_{4}, \mathrm{MeOH}$, reflux, 8 h; (ii) $\mathrm{NH}_{2} \mathrm{NH}_{2} \cdot \mathrm{H}_{2} \mathrm{O}, \mathrm{EtOH}$, reflux, $3 \mathrm{~h}$.

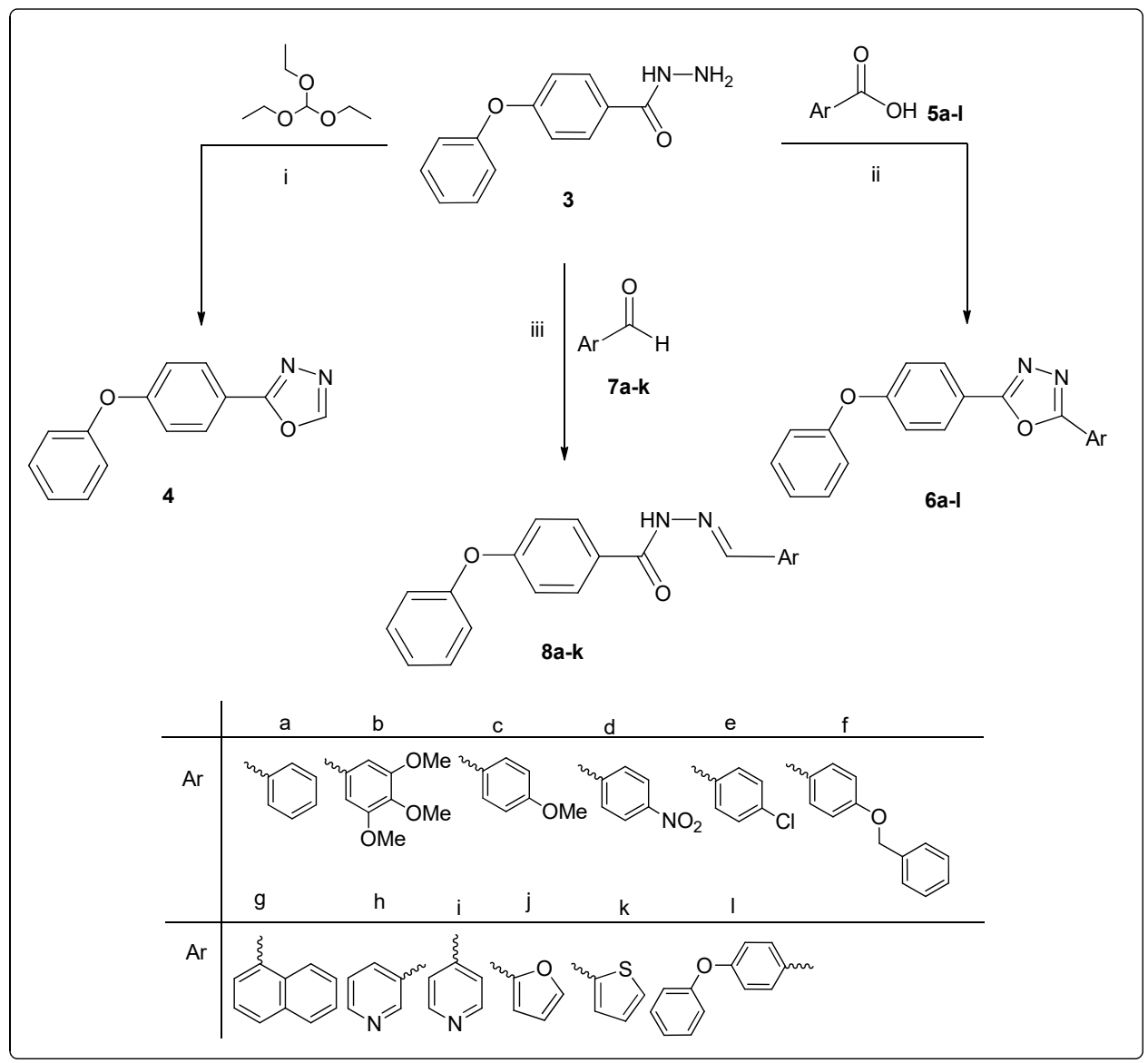

Scheme 2. Reagents and conditions: (i) Triethyl orthoformate reflux, $3 \mathrm{~h}$; (ii) $\mathrm{POCl}_{3}$, reflux, 2-3 h; (iii) EtOH, reflux, 3-6 h. 


\subsection{Biology}

\subsubsection{Anti-Tubercular Activity}

Compounds 3, 4, 6a-1 and $\mathbf{8 a - k}$ were evaluated for antitubercular activity against Mycobacterium tuberculosis H37Rv using the standard Microplate Alamar Blue Assay (MABA) protocol (Table 1) [31,32]. The MIC reported as the lowest concentration $(\mu \mathrm{g} / \mathrm{mL})$ of targets inhibited the growth of an organism. Triclosan and INH were used as reference standards. Among the tested compounds, the results displayed that most of them i.e., 3, 4, $\mathbf{6 h}, \mathbf{6 i}, \mathbf{6 1}, \mathbf{8 g}$, and $\mathbf{8 k}$ showed promising anti-tubercular activity (MIC, $0.61-1.69 \mu \mathrm{g} / \mathrm{mL}$ ) in comparison to triclosan (MIC, $10 \mu \mathrm{g} / \mathrm{mL}$ ) and INH (MIC, $0.2 \mu \mathrm{g} / \mathrm{mL}$ ).

Table 1. The antitubercular activity of the diphenyl ether derivatives against H37Rv strain of M. tuberculosis (MIC $(\mu \mathrm{g} / \mathrm{mL})$ and their inhibition for InhA reductase enzyme $\left(\mathrm{IC}_{50} \mu \mathrm{M}\right)$.

\begin{tabular}{|c|c|c|c|c|c|c|}
\hline $\mathrm{Ar}$ & No. & MIC $(\mu \mathrm{g} / \mathrm{mL})$ & $\mathrm{IC}_{50}(\mu \mathrm{M})$ & No. & $\operatorname{MIC}(\mu \mathrm{g} / \mathrm{mL})$ & $\mathrm{IC}_{50}(\mu \mathrm{M})$ \\
\hline & 3 & 0.86 & 3.43 & 4 & 0.61 & 3.28 \\
\hline & $6 a$ & 2.74 & $>100$ & $8 a$ & 34.54 & 73.23 \\
\hline & $6 b$ & 34.64 & $>100$ & $8 b$ & 50.20 & $>100$ \\
\hline & $6 c$ & 39.04 & $>100$ & $8 c$ & 20.68 & 75.46 \\
\hline & $6 d$ & 15.15 & 68.44 & $8 d$ & 7.67 & 19.75 \\
\hline & $6 e$ & 15.89 & 64.55 & $8 e$ & 2.89 & 7.46 \\
\hline & $6 f$ & 8.23 & 41.24 & $8 f$ & 22.62 & 29.89 \\
\hline & $6 \mathrm{~g}$ & 12.23 & 33.82 & $8 g$ & 0.98 & 4.23 \\
\hline & $6 \mathrm{~h}$ & 1.40 & 5.08 & $8 \mathrm{~h}$ & 6.96 & 26.54 \\
\hline & $6 i$ & 1.40 & 11.72 & $8 \mathbf{i}$ & 6.82 & 21.39 \\
\hline & $6 j$ & 11.12 & 51.42 & $8 j$ & 6.35 & 18.37 \\
\hline & $6 k$ & 3.65 & 78.65 & $8 k$ & 1.69 & 5.61 \\
\hline & 61 & 1.17 & 5.63 & INH & 0.20 & ND \\
\hline & - - - - & - & - & Triclosan & 10.00 & 1.10 \\
\hline
\end{tabular}

The structure-activity relationship acquired suggested that installing the open-chain oxadiazole moiety into the diphenyl ether caused a remarkable increase in activity as seen in compound 3 (MIC, $0.86 \mu \mathrm{g} / \mathrm{mL}$ ). Interestingly, the cyclic and unsubstituted oxadiazole 4 (MIC, $0.61 \mu \mathrm{g} / \mathrm{mL}$ ) showed experienced and superior activity than all the 5-substituted analogs. Noteworthy, the 5-phenyl 6a and 5-benzyloxy-phenyl 6f derivatives experienced a slight decrease in activity with MIC of 2.74, $8.23 \mu \mathrm{g} / \mathrm{mL}$, respectively. It was noted, however, that replacement of the phenyl group at the oxadiazole ring with a six-membered heterocyclic system showed a slight improvement in the activity as depicted in compounds $6 \mathrm{~h}$ and $6 \mathbf{i}(\mathrm{MIC}, 1.40 \mu \mathrm{g} / \mathrm{mL}$ ) while alteration of this ring into 5-membered one exhibited less activity such as 6k (MIC, $3.65 \mu \mathrm{g} / \mathrm{mL}$ ). Expectedly, bis(diphenylether) oxadiazole 61 was the most active in this 5 -substituted series $(1.17 \mu \mathrm{g} / \mathrm{mL})$ that may be due to the incorporation of extra phenyl 
ether nucleus to the backbone. The substitutions on the 5-aryl groups have a deleterious effect on biological activity especially the activating methoxy substituents in compounds $\mathbf{6 b}$ and $\mathbf{6 c}$.

In a related vein, the arylidene group of compounds $\mathbf{8 a}-\mathbf{k}$ was found comparably less active in vitro where this situation may modify in vivo, as we know arylidenes are prodrugs. By comparing these derivatives, it is clear that the introduction of the heterocyclic system into the diphenyl ether hydrazone structure either five, compound $\mathbf{8 j}$ (MIC, $6.35 \mu \mathrm{g} / \mathrm{mL}$ ) or six-membered ring system, compounds $\mathbf{8 h}-\mathbf{i}(\mathrm{MIC} \sim 6.90 \mu \mathrm{g} / \mathrm{mL})$ caused a decrease in the anti-tubercular activity. Similarly, replacement of the heterocyclic ring with substituted phenyl group i.e., compounds $\mathbf{8 d}(\mathrm{MIC}, 7.67 \mu \mathrm{g} / \mathrm{mL}$ ) and $\mathbf{8 e}$ (MIC, $2.89 \mu \mathrm{g} / \mathrm{mL}$ ) showed weak inhibition. Surprisingly, a remarkable increase in the activity was observed for compounds $\mathbf{8 g}(0.98 \mu \mathrm{g} / \mathrm{mL}$; Ar = Napthyl) and 8k (MIC, $1.69 \mu \mathrm{g} / \mathrm{mL}$; Ar = thienyl). Generally, the order of activity against MTB strain H37Rv of diphenyl ether bearing arylidene core was Ar $=$ zero $>$ naphthyl $>$ thienyl $>p$-Cl-phenyl $>3$ pyridinyl $\sim 4$-pyridinyl $>p$ - $\mathrm{NO}_{2}$-phenyl. All these data suggested that the diaryl ether scaffold is very important for the antitubercular activity, removal of the $o$-chloro group in triclosan, as well as the introduction of oxadiazole or its open-chain analog hydrazide, increased activity than the parent triclosan. In conclusion, compounds 3, 4, 6h, 6i, 61, 8g, and 8k showed higher antitubercular activity than the reference standard triclosan. Three potential lead candidates $\mathbf{3}$ and $\mathbf{4}$ and $\mathbf{8 g}$ were found to be the most active in which hydrazide oxadiazole or naphthyl arylidine moieties are directly attached to an unsubstituted diphenyl ether core. Their structural features could be further investigated for their potential use as new anti-TB drugs.

\subsubsection{In Vitro InhA Reductase Enzyme Inhibition}

The in vitro inhibitions data of the target compounds for the InhA reductase enzyme are presented as $\mathrm{IC}_{50} \mu \mathrm{M}$ values in Table 1 . Among the tested compounds, the highly active hydrazide 3 and oxadiazole 4 and naphthyl arylidine $8 \mathrm{~g}$ showed the highest inhibition of InhA reductase with $\mathrm{IC}_{50}$ of $3.43 \mu \mathrm{M}$ and $3.28 \mu \mathrm{M}$ and $4.23 \mu \mathrm{M}$, respectively, but still less than the reference triclosan $\left(\mathrm{IC}_{50}, 1.10 \mu \mathrm{M}\right)$. On the other hand, oxadiazole derivatives $\mathbf{6 a} \mathbf{a}-\mathbf{c}$ and the corresponding arylidenes $\mathbf{8 a}-\mathbf{c}$ showed deleterious activity as inhibitors for InhA reductase. The oxadiazoles $\mathbf{6 h}, \mathbf{6 1}$ and arylidine $\mathbf{8 k}$ ether derivatives experienced good inhibition activities with $\mathrm{IC}_{50}$ of 5.08, 5.63, and 5.61, respectively. In general, most of the tested target compounds showed good inhibition for InhA reductase. Besides, other compounds show promising activity against H37Rv strain of $M$. tuberculosis. We believe, additional modes of mechanisms involved in the activity.

Moreover, calculated results showed a slight correlation between the molecular weight, Wiener index, and topological diameter to biological activity with $\mathrm{R}=0.308,0.315,0.259$, respectively. These results indicate the importance of the overall volume of a molecule on the biological activity, but it is not the only factor affecting biological activity. On the other hand, it was found that all the thermodynamic parameters (i.e., molar refractivity and Gibbs free energy and heat formation) have a negative correlation with the biological activity where $\mathrm{R}=-0.100,-0.602$ and -0.683 , respectively (Table 2).

The molecules that display a negative sign for the heat of formation parameter means that binding of these molecules with their protein targets may be spontaneous and do not require energy. The protein-ligand binding occurs only when the change in Gibbs free energy ( $\Delta G$ : is the free energy that gives an indication about a binding affinity to the target site) of the system is negative. Furthermore, Hydrogen bonding is one of the important factors which is responsible for determining the three-dimensional structure of folded proteins including enzymes and the ligand molecule. Also, it is involved in determining the polarity and permeability of compounds. Calculations showed that antimycobacterial activity expressed in MIC $(\mu \mathrm{g} / \mathrm{mL})$ is negatively correlated with both hydrogen bond donor and hydrogen bond acceptor count. Calculation of the number of hydrogen bond donors, hydrogen bond acceptors, and other parameters of Lipinski's (rule of five) showed that most of the target compounds presented drug-like characters [33-35]. 
Table 2. Correlation between calculated physicochemical parameters and biological activity.

\begin{tabular}{|c|c|c|c|c|c|c|c|c|c|c|c|c|}
\hline $\begin{array}{l}\text { Comp. } \\
\text { No. }\end{array}$ & $\underset{(\mu \mathrm{g} / \mathrm{mL})}{\mathrm{MIC}}$ & $\begin{array}{c}\text { Molecular } \\
\text { Mass }\end{array}$ & $\begin{array}{l}\text { Balaban } \\
\text { Index }\end{array}$ & $\begin{array}{c}\text { Molecular } \\
\text { Topology: } \\
\text { Wiener Index }\end{array}$ & $\begin{array}{c}\text { Topological } \\
\text { Diameter } \\
\text { Bond (s) }\end{array}$ & $\begin{array}{c}\text { Molar } \\
\text { Refractivity, } \\
\mathrm{cm}^{3} / \mathrm{mol}\end{array}$ & $C \log p$ & $\begin{array}{c}\text { Heat of } \\
\text { Formation } \\
{[\mathrm{kJ} / \mathrm{mol}]}\end{array}$ & $\begin{array}{c}\text { Gibbs Energy } \\
{[\mathrm{kJ} / \mathrm{mol}]} \\
\text { Energy }\end{array}$ & $\begin{array}{l}\text { H Bond } \\
\text { Donor }\end{array}$ & $\begin{array}{l}\text { H Bond } \\
\text { Acceptor }\end{array}$ & $\begin{array}{l}\text { Lipinski's } \\
\text { Rule }\end{array}$ \\
\hline 3 & 0.86 & 228.250 & 118,297 & 586 & 11 & 6.589 & 2.231 & -60.70 & 176.14 & 2 & 3 & Yes \\
\hline 4 & 0.61 & 238.250 & 121,561 & 682 & 11 & 6.656 & 2.753 & 115.57 & 339.72 & 0 & 4 & Yes \\
\hline $6 a$ & 2.74 & 314.340 & 400,250 & 1561 & 15 & 9.167 & 4.806 & 216.79 & 493.02 & 0 & 4 & Yes \\
\hline $6 b$ & 34.64 & 404.422 & 110,779 & 2828 & 17 & 11.018 & 4.427 & -276.20 & 174.39 & 0 & 7 & Yes \\
\hline $6 \mathrm{c}$ & 39.04 & 344.370 & 59,322 & 1990 & 17 & 9.784 & 4.679 & 52.46 & 386.81 & 0 & 5 & Yes \\
\hline $6 \mathrm{~d}$ & 15.15 & 359.340 & 71,066 & 2219 & 17 & 9.778 & ND & ND & ND & 0 & 5 & Yes \\
\hline $6 e$ & 15.89 & 348.770 & 488,162 & 1763 & 16 & 9.659 & 5.364 & 189.58 & 471.46 & 0 & 4 & No \\
\hline $6 f$ & 8.23 & 420.470 & 142,971 & 3769 & 21 & 12.295 & 6.412 & 165.15 & 549.74 & 0 & 5 & No \\
\hline $6 \mathrm{~g}$ & 12.23 & 364.404 & 706,482 & 2391 & 17 & 10.855 & 5.803 & 313.83 & 623.72 & 0 & 4 & No \\
\hline $6 \mathrm{~h}$ & 1.40 & 315.332 & 400,250 & 1561 & 15 & 8.956 & 3.468 & 270.22 & 561.65 & 0 & 5 & No \\
\hline $6 \mathbf{i}$ & 1.40 & 315.332 & 400,250 & 1561 & 15 & 8.956 & 3.468 & 270.22 & 561.65 & 0 & 5 & Yes \\
\hline $6 j$ & 11.12 & 304.305 & 326,596 & 1380 & 14 & 8.381 & 3.421 & 74.450 & 372.20 & 0 & 5 & Yes \\
\hline $6 \mathrm{k}$ & 3.65 & 320.062 & 32,659 & 1380 & 14 & 8.976 & 4.788 & 251.71 & 498.18 & 0 & 4 & Yes \\
\hline 61 & 1.17 & 406.440 & 120,686 & 3377 & 20 & 11.831 & 6.343 & 185.97 & 541.32 & 0 & 5 & No \\
\hline $8 a$ & 34.54 & 384.390 & 104,520 & 2849 & 19 & 10.994 & 4.734 & 145.52 & ND & 1 & 6 & Yes \\
\hline $8 b$ & 50.20 & 474.470 & 250,732 & 4753 & 21 & 12.845 & 4.354 & -347.47 & ND & 1 & 9 & Yes \\
\hline $8 c$ & 20.68 & 414.420 & 145,706 & 3497 & 21 & 11.611 & 4.607 & -18.810 & ND & 1 & 7 & Yes \\
\hline $8 d$ & 7.67 & 414.420 & 145,706 & 3497 & 21 & 11.610 & ND & -18.81 & ND & 1 & 7 & Yes \\
\hline $8 e$ & 2.89 & 418.840 & 12,360 & 3158 & 20 & 11.485 & 5.292 & 118.31 & ND & 1 & 6 & Yes \\
\hline $8 \mathrm{f}$ & 22.62 & 490.519 & 301,096 & 6023 & 25 & 14.122 & 6.339 & 93.88 & ND & 1 & 7 & No \\
\hline $8 g$ & 1.40 & 434.450 & 161,770 & 4015 & 20 & 12.682 & 5.730 & 242.56 & ND & 1 & 6 & No \\
\hline $8 \mathrm{~h}$ & 6.96 & 385.380 & $1,045,201$ & 2849 & 19 & 10783 & 3.396 & 198.95 & ND & 1 & 7 & No \\
\hline $8 i$ & 6.82 & 385.383 & $1,045,201$ & 2849 & 19 & 10.783 & 3.396 & 198.95 & ND & 1 & 7 & Yes \\
\hline $8 j$ & 6.35 & 374.356 & 880,346 & 2566 & 18 & 10.208 & 3.349 & 3.180 & ND & 1 & 7 & Yes \\
\hline $8 k$ & 1.69 & 390.417 & 88,034 & 2566 & 18 & 10.803 & 4.715 & 180.44 & ND & 1 & 6 & Yes \\
\hline $\mathbf{R}$ & - & 0.308 & -0.179 & 0.315 & 0.260 & -0.100 & 0.152 & -0.683 & -0.602 & -0.370 & -0.360 & - \\
\hline
\end{tabular}




\subsubsection{Cytotoxic Study}

Compounds 3, 4, 6h, 6i, 6l, 8g, and 8k displaying the lowest MIC values were selected for cytotoxicity test using model eukaryotic cell line (Vero) at the concentration range from 0.1 to $200 \mu \mathrm{g} / \mathrm{mL}$ with $\mathrm{IC}_{50}$ values more than $60 \mu \mathrm{g} / \mathrm{mL}$. Compounds did not show a high cytotoxic effect on Vero cells (Table 3).

Table 3. MIC, $\mathrm{IC}_{50}$, and SI values determined for the selected most promising compounds.

\begin{tabular}{cccc}
\hline \multirow{2}{*}{ Compound } & M. tuberculosis & Vero Cell Line & \multirow{2}{*}{ SI (IC $\left._{50} / \mathbf{M I C}\right)$} \\
\cline { 2 - 3 } & MIC, $\boldsymbol{\mu g} / \mathbf{m L}$ & IC $_{\mathbf{5 0}} \boldsymbol{\mu g} / \mathbf{m L} \pm \mathbf{S E M}$ & \\
\hline $\mathbf{3}$ & 0.86 & $80.7 \pm 1.31$ & 93.83 \\
$\mathbf{4}$ & 0.61 & $99.4 \pm 1.79$ & 162.95 \\
$\mathbf{6 h}$ & 1.40 & $91.4 \pm 1.55$ & 65.29 \\
$\mathbf{6 i}$ & 1.40 & $95.5 \pm 1.36$ & 68.21 \\
$\mathbf{6 1}$ & 1.17 & $92.6 \pm 1.80$ & 79.15 \\
$\mathbf{8 g}$ & 0.98 & $72.2 \pm 1.87$ & 73.67 \\
$\mathbf{6 k}$ & 1.69 & $68.6 \pm 1.19$ & 40.59 \\
\hline
\end{tabular}

Notably, compound 4 which showed the lowest MIC value of $0.61 \mu \mathrm{g} / \mathrm{mL}$ also displayed very low cytotoxicity to Vero cells at all tested concentrations $(0.1-200 \mu \mathrm{g} / \mathrm{mL})$ with $\mathrm{IC}_{50}$ values close to $100 \mu \mathrm{g} / \mathrm{mL}$ (99.4) and accordingly its selectivity index (SI) value more than 10 (162.95), providing its promising potential in biomedical application. In the case of compounds $3, \mathbf{6 h}, \mathbf{6} \mathbf{i}$, and $\mathbf{6 1}$, a slight reduction in cell viability was observed with $\mathrm{IC}_{50}$ ranging from $80.7-95.5 \mu \mathrm{g} / \mathrm{mL}$, whereas $\mathbf{8 g}$ and $8 \mathbf{k}$ showed the more pronounced cytotoxic effect was with $\mathrm{IC}_{50} 72.3$ and $68.6 \mu \mathrm{g} / \mathrm{mL}$, respectively. Therefore, it may be assumed that $4,6 \mathbf{h}, \mathbf{6 i}, \mathbf{6 1}, \mathbf{8 g}$, and $8 \mathrm{k}$ derivatives, which were non-toxic or showed very low toxicity at tested concentration range, are very promising and potentially safe antimycobacterial compounds.

\subsubsection{Molecular Docking}

The inhibitory profile of triclosan and compounds 3, $8 \mathrm{~g}$ (models for the higher inhibitory activity) and $\mathbf{8 b}$ (model for the deleterious inhibitory activity) were chosen for further investigation by docking into InhA reductase active site (PDB code: $3 \mathrm{FNH}$ ) to gain insight into the potential binding modes [36-38]. As a first step, for the validation of docking parameters, the co-crystal ligand (JPJ400) was re-docked at the catalytic site of the protein. The RMSD between co-crystal and re-docked poses was found to be $0.331(<2 \mathrm{~A})$, which confirmed the validity of the docking parameters. Triclosan exploited two major interactions. Firstly, the hydrophilic interaction has been noticed by the hydroxyl OH via two hydrogen bonds i.e., the oxygen engaged with the hydrophilic side chainTyr158 while the hydrogen forms the second hydrogen bond with NAD. Secondly, clusters of hydrophobic interactions including pi-or thirteen—alkyl interactions with NAD, Phe97, Met161, Pro193, Ala198, Met199, and Ile202 were also found. Further, pi-cation interaction with NAD, pi-sulfur interaction with Met103 and also, pi-pi stacked interaction with Phe149 have been noticed as well (Figure 3).

By careful analysis of the docked poses of compounds $\mathbf{3}$ and $\mathbf{8 g}$, it was clear that both compounds can occupy the same binding mode as Triclosan with binding interaction energy $(-24.264) \mathrm{kcal} / \mathrm{mol}$ (for compound 3) to $-17.148 \mathrm{kcal} / \mathrm{mol}$ (for compound $8 \mathrm{~g}$ ) compared to Triclosan $(-22.507) \mathrm{kcal} / \mathrm{mol}$. The hydrophilic interaction has been noticed in compound 3 via the carbonyl oxygen that forms a hydrogen bond with Tyr158 side chain, while the hydrogen of amino group forms the second one with NAD, but compound $8 \mathrm{~g}$ was involved in only one i.e., $\mathrm{N} 4$ of the oxadiazole ring forms one hydrogen bond with the hydrophilic residue Tyr 158, likely due to its bulkiness and this may explain its low potency than compound 3 (Figures 4 and 5). The hydrophobic interactions in compound 3 were incorporating three pi-alkyl interactions with Pro193, Met199 and Leu218. Moreover, two pi-sulfur interactions with Met155 and Met199, one attractive charge interaction with NAD and two pi-pi T-shaped interactions with Phe149 and Tyr158 have been observed. In the same mode, compound $\mathbf{8 g}$ 
displays its hydrophobic interactions with different amino acid residues that embodying five pi-pi stacked interactions with NAD, Phe97, and Phe149. Also, eight pi-alkyl interactions with Phe149, Met161, Ala198, Ala201, Ile215, and Leu218 were found.
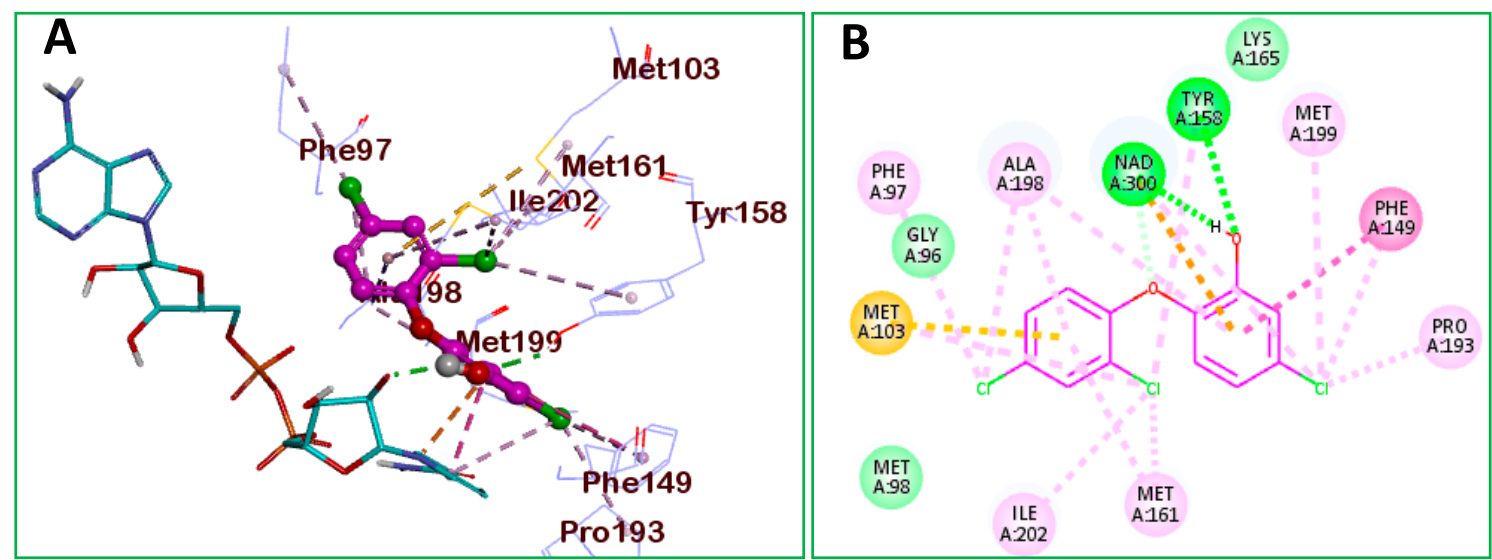

Figure 3. Docking and binding mode of Triclosan into the active site of InhA reductase enzyme (PDB code: 3FNH). (A) 3D structure of triclosan (pink), (B) 2D structure of Triclosan (pink).

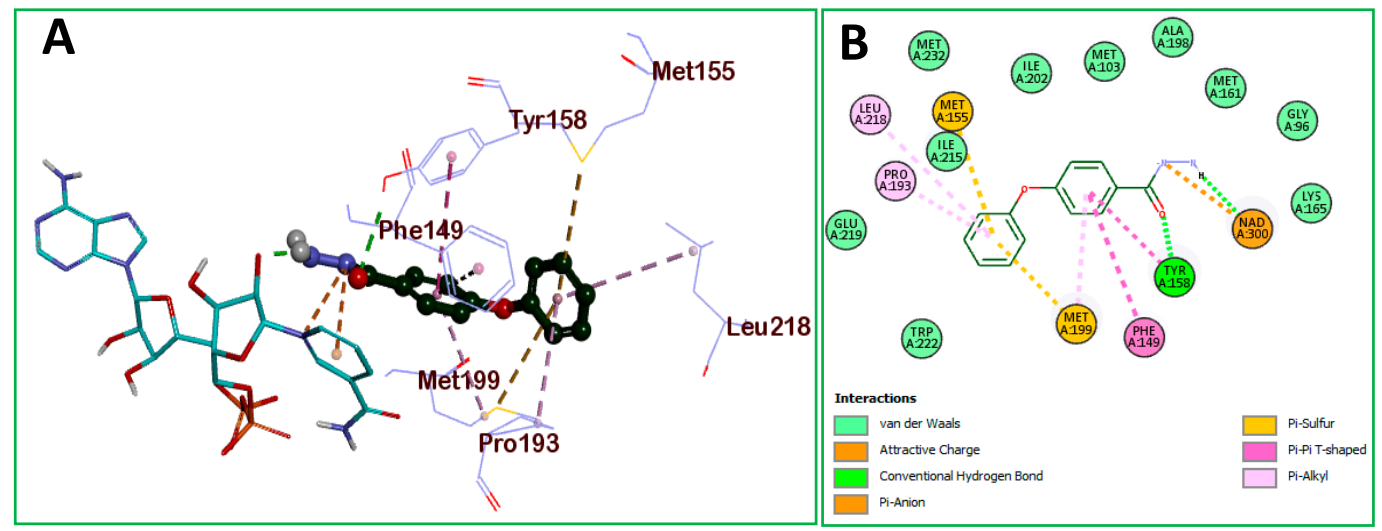

Figure 4. Docking and binding mode of compound 3 into the active site of InhA reductase enzyme (PDB code: 3FNH). (A) 3D structure of compound 3 (green), (B) 2D structure of compound 3 (green).
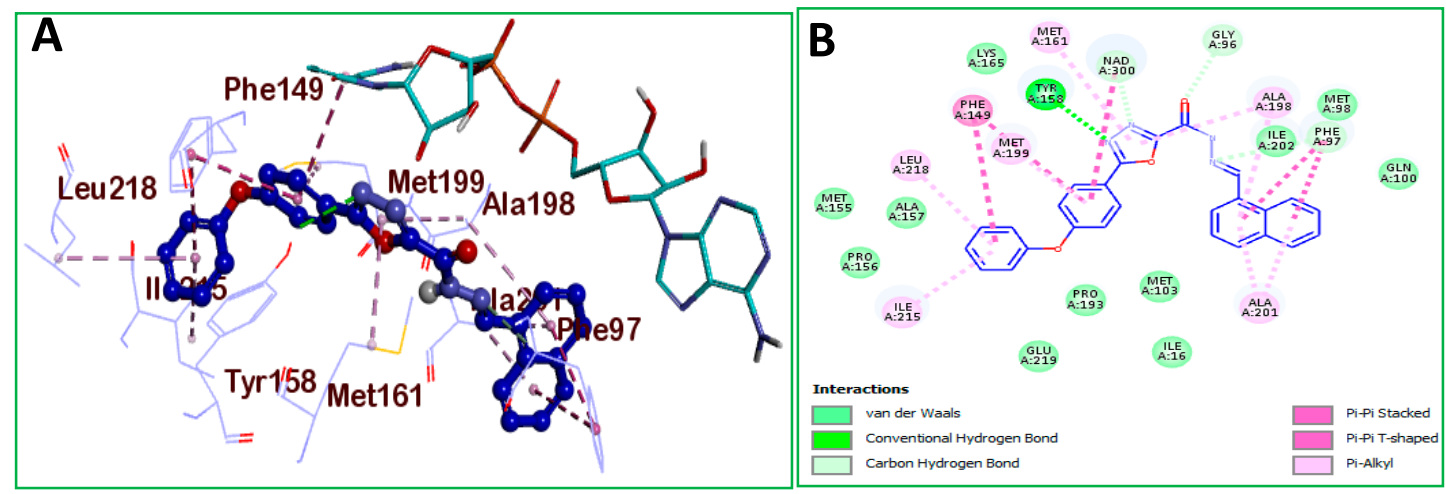

Figure 5. Docking and binding mode of compound $8 \mathrm{~g}$ into the active site of InhA reductase enzyme (PDB code: 3FNH). (A) 3D structure of compound $8 \mathrm{~g}$ (blue), (B) 2D structure of compound $8 \mathrm{~g}$ (blue).

It is worth pointing out that in compound $\mathbf{8 b}$, the hydrogen of the hydrazide group showed unfavorable donor-donor interaction (red color) with binding interaction energy $(+11.145) \mathrm{kcal} / \mathrm{mol}$. 
Also, the compound has not involved in any hydrogen bonds and forms only hydrophobic interaction with some amino acid residues and this may reflect the low activity of the compound (Figure 6).
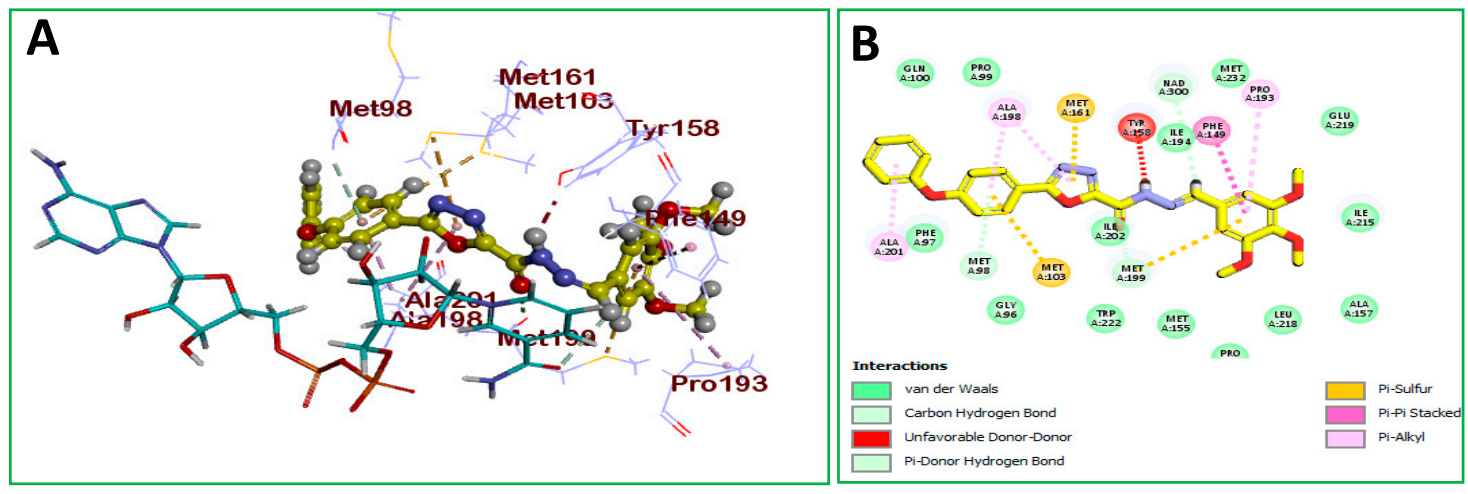

Figure 6. Docking and binding mode of compound $\mathbf{8 b}$ into the active site of the InhA reductase enzyme (PDB code: 3FNH). (A) 3D structure of compound $\mathbf{8 b}$ (yellow), (B) 2D structure of compound $\mathbf{8 b}$ (yellow).

\section{Material and Methods}

\subsection{Chemistry}

Melting points were recorded on a Stuart SMP30 (Stuart, Cole-Parmer, Staffordshire, UK) melting point apparatus. IR spectra $\left(\mathrm{KBr}, \mathrm{cm}^{-1}\right)$ were recorded on a Vector 22FT-IR Fourier Transform infrared (FTIR) spectrometer (Bruker, Billerica, MA, USA). ${ }^{1} \mathrm{H}-\mathrm{NMR}$ and ${ }^{13} \mathrm{C}-\mathrm{NMR}$ spectra were recorded at 500 and $125 \mathrm{MHz}$, respectively, on an Inova $500 \mathrm{MHz}$ spectrometer (Bruker, Billerica, MA, USA), using dimethyl sulphoxide (DMSO- $\left.\mathrm{d}_{6}\right) /$ deuterated chloroform $\left(\mathrm{CDCl}_{3}\right)$ as a solvent and tetramethylsilane (TMS) as an internal standard (chemical shift in $\delta, \mathrm{ppm}$ ). Mass spectra were determined using a Shimadzu GC/MS QP 1000 EX system (Shimadzu Corporation, Tokyo, Japan) with ionization energy $70 \mathrm{eV}$. Elemental analysis were determined using an Automatic Elemental Analyzer CHN Model 2400 (Perkin Elmer, Richmond, CA, USA) at the Micro-analytical Center, Faculty of Science, Cairo University (Cairo, Egypt). All the results of elemental analyses corresponded to the calculated values within experimental error. Progress of the reaction was monitored by thin-layer chromatography (TLC) using precoated TLC sheets with ultraviolet (UV) fluorescent silica gel (60F254, Merck, Kenilworth, NJ, USA) and spots were visualized by iodine vapors or irradiation with UV light $(254 \mathrm{~nm})$. All the chemicals were purchased from Sigma-Aldrich Chemicals, St. Louis, MO, USA. Compounds 2, 3, 4, 6g, 6i-k and $\mathbf{8 i -} \mathbf{k}$ were prepared according to the reported procedures $[30,39,40]$.

Methyl 4-phenoxybenzoate (2). Concentrated $\mathrm{H}_{2} \mathrm{SO}_{4}(1.0 \mathrm{~mL})$ was added dropwise to a solution of 4-phenoxybenzoic acid $(3.3 \mathrm{~g}, 15.6 \mathrm{mmol})$ in methanol $(25 \mathrm{~mL})$. The reaction mixture was allowed to reflux for $8 \mathrm{~h}$. The reaction mixture was concentrated under reduced pressure. The obtained residue was treated with $10 \%$ aqueous $\mathrm{NaHCO}_{3}(10 \mathrm{~mL})$. Stirring was continued for $15 \mathrm{~m}$. During which time, the precipitate was formed then collected by filtration then dried and purified by crystallization using methanol to afford white solid in $80 \%$ yield; m.p. $159-160{ }^{\circ} \mathrm{C} ;{ }^{1} \mathrm{H}-\mathrm{NMR}\left(\mathrm{CDCl}_{3}\right) \delta: 3.90\left(\mathrm{~s}, 3 \mathrm{H}, \mathrm{OCH}_{3}\right)$, $6.99(\mathrm{~d}, J=8.8 \mathrm{~Hz}, 2 \mathrm{H}, \mathrm{ArH}), 7.07(\mathrm{~d}, J=7.8 \mathrm{~Hz}, 2 \mathrm{H}, \mathrm{ArH}), 7.19(\mathrm{t}, J=7.9 \mathrm{~Hz}, 1 \mathrm{H}, \mathrm{ArH}), 7.39(\mathrm{t}, 2 \mathrm{H}$, $J=7.9 \mathrm{~Hz}, \mathrm{ArH}), 8.00(\mathrm{~d}, J=8.4 \mathrm{~Hz}, 2 \mathrm{H}, \mathrm{ArH}) ;{ }^{13} \mathrm{C}-\mathrm{NMR}\left(\mathrm{CDCl}_{3}\right) \delta: 52.1\left(\mathrm{OCH}_{3}\right), 117.4(\mathrm{C} 3, \mathrm{C} 5), 120.2$ $\left(\mathrm{C} 3^{\prime}, \mathrm{C}^{\prime}\right), 124.6(\mathrm{C} 1), 130.2\left(\mathrm{C}^{\prime}, \mathrm{C} 6^{\prime}\right), 131.8(\mathrm{C} 2, \mathrm{C} 6), 155.8(\mathrm{C} 4), 162.0\left(\mathrm{C}^{\prime}\right), 166.7\left(\mathrm{C} 4^{\prime}\right), 207.1(\mathrm{C}=\mathrm{O})$.

4-Phenoxybenzohydrazide (3). Methyl 4-phenoxybenzoate $(228 \mathrm{mg}, 1 \mathrm{mmol})$ was added to a solution of hydrazine hydrate $98 \%(0.5 \mathrm{~mL})$ in ethanol $(5 \mathrm{~mL})$. The mixture was refluxed for $2 \mathrm{~h}$. The reaction mixture was evaporated under vacuum, water $(10 \mathrm{~mL})$ was added to the obtained residue. The obtained mixture was extracted with ethyl acetate $(2 \times 10 \mathrm{~mL})$. The combined organic layers were then washed with a saturated solution of sodium bicarbonate and $(1 \times 10 \mathrm{~mL})$ then brine $(1 \times 10 \mathrm{~mL})$ before being 
dried $\left(\mathrm{MgSO}_{4}\right)$. The obtained filtrate was concentrated under reduced pressure, filtered off and the precipitate was subjected to crystallization using EtOAc/petroleum ether. White solid, Yield: 84\%; m.p.: 180-190 ${ }^{\circ} \mathrm{C} ;{ }^{1} \mathrm{H}-\mathrm{NMR}\left(\mathrm{CDCl}_{3}\right) \delta: 4.49\left(\mathrm{~s}, 2 \mathrm{H}, \mathrm{NH}_{2}\right), 7.00(\mathrm{~d}, J=8.0 \mathrm{~Hz}, 2 \mathrm{H}, \mathrm{ArH}), 7.05(\mathrm{~d}, J=8.0 \mathrm{~Hz}, 2 \mathrm{H}$, $\operatorname{ArH}), 7.18(\mathrm{t}, J=7.5 \mathrm{~Hz}, 1 \mathrm{H}, \mathrm{ArH}), 7.36-7.40(\mathrm{~m}, 2 \mathrm{H}, \mathrm{ArH}), 7.41(\mathrm{~s}, 1 \mathrm{H}, \mathrm{NH}), 7.72(\mathrm{~d}, J=8.2 \mathrm{~Hz}, 2 \mathrm{H}$, ArH). ${ }^{13} \mathrm{C}-\mathrm{NMR}\left(\mathrm{CDCl}_{3}\right)$ 8: $118.0(\mathrm{C} 3, \mathrm{C} 5), 120.1\left(\mathrm{C}^{\prime}, \mathrm{C}^{\prime}\right), 124.6(\mathrm{C} 1), 127.1\left(\mathrm{C}^{\prime}\right), 128.9\left(\mathrm{C}^{\prime}, \mathrm{C}^{\prime}\right)$, 130.2 (C2, C6), 155.9 (C4), $161.1\left(\mathrm{C}^{\prime}\right), 168.3(\mathrm{C}=\mathrm{O})$.

2-(4-Phenoxyphenyl)-1,3,4-oxadiazole (4). A mixture of hydrazide 3 (1 mmol) and triethyl orthoformate $(2 \mathrm{~mL})$ were refluxed for $3 \mathrm{~h}$. The reaction mixture was concentrated under vacuum; the residue was cooled and then treated with hexane $(5 \mathrm{~mL})$. The obtained precipitate was filtered off and recrystallized from ethanol/water (1:1). White solid, Yield: 73\%; m.p. 140-144 ${ }^{\circ} \mathrm{C}[26] .{ }^{1} \mathrm{H}-\mathrm{NMR}$ (DMSO- $\left.d_{6}\right) \delta$ : 7.14-7.17 (m, 4H, ArH), 7.25 (t, J = 7.5 Hz, 1H ArH), 7.45-7.49 (m, 2H, ArH), 8.01-8.04 (m, 2H, ArH), 9.31 (s, 1H, CH-oxadiazole). ${ }^{13} \mathrm{C}-\mathrm{NMR}$ (DMSO- $d_{6}$ ) $\delta: 117.8$ (C3, C5), 118.3 (C3', $\left.\mathrm{C}^{\prime}\right), 119.9$ (C1), 124.7 $\left(\mathrm{C1}^{\prime}\right), 128.9(\mathrm{C} 2, \mathrm{C} 6), 130.3\left(\mathrm{C}^{\prime}, \mathrm{C}^{\prime}\right), 154.2\left(\mathrm{C} 4^{\prime}\right), 155.1(\mathrm{C} 4), 160.2(\mathrm{C}=\mathrm{N}), 163.3(\mathrm{C}=\mathrm{N})$. Anal. Calcd. for $\mathrm{C}_{14} \mathrm{H}_{10} \mathrm{~N}_{2} \mathrm{O}_{2}$ : C, 70.58; H, 4.23; N, 11.76. Found: C, 70.50; H, 4.48; N 11.37.

2-(4-Phenoxyphenyl)-5-phenyl-1,3,4-oxadiazole (6a). White solid, Yield; 70\%; m.p. 233-235 ${ }^{\circ} \mathrm{C} .{ }^{1} \mathrm{H}-\mathrm{NMR}$ $\left(\mathrm{CDCl}_{3}\right)$ 8: 7.01-7.07 (m, 5H, ArH), 7.18-7.22 (m, 1H, ArH), 7.38-7.56 (m, 5H, ArH), 7.85-7.88 (m, 3H, ArH). ${ }^{13} \mathrm{C}-\mathrm{NMR}\left(\mathrm{CDCl}_{3}\right)$ 8: 118.0 (C3, C5), 119.1 (C3', C5'), 120.3 (C3"), 122.9 (C5"), 124.8 (C1), 125.7 (C1', C1"), 127.5 (C2, C6), 129.1 (C2', C6'), 129.5 (C2"), 130.3 (C6"), 131.6 (C4), 132.7 (C4'), 161.7, (C4"), $164.3(\mathrm{C}=\mathrm{N}), 164.5(\mathrm{C}=\mathrm{N})$. Anal. Calcd for $\mathrm{C}_{20} \mathrm{H}_{14} \mathrm{~N}_{2} \mathrm{O}_{2}: \mathrm{C}, 76.42 ; \mathrm{H}, 4.49 ; \mathrm{N}, 8.91$. Found: $\mathrm{C}, 76.54 ; \mathrm{H}$, $4.23 ; \mathrm{N} 9.27$.

2-(4-Phenoxyphenyl)-5-(3,4,5-trimethoxyphenyl)-1,3,4-oxadiazole (6b). White solid, Yield; 76\%, m.p. 250-253 ${ }^{\circ} \mathrm{C} .{ }^{1} \mathrm{H}-\mathrm{NMR}\left(\mathrm{CDCl}_{3}\right)$ 8: $3.92(\mathrm{~s}, 3 \mathrm{H}, \mathrm{OMe}), 3.97(\mathrm{~s}, 6 \mathrm{H}, 2 \mathrm{OMe}), 7.05(\mathrm{~d}, J=8.0 \mathrm{~Hz}, 2 \mathrm{H}, \mathrm{ArH})$, 7.18-7.24 (m, 3H, ArH), $7.34(\mathrm{~s}, 2 \mathrm{H}, \mathrm{ArH}), 7.64-7.70(\mathrm{~m}, 2 \mathrm{H}, \mathrm{ArH}), 7.98(\mathrm{~d}, J=8.2 \mathrm{~Hz}, 2 \mathrm{H}, \mathrm{ArH})$. ${ }^{13} \mathrm{C}-\mathrm{NMR}\left(\mathrm{CDCl}_{3}\right)$ 8: $56.7(\mathrm{OMe}), 56.8(\mathrm{OMe}), 61.3(\mathrm{OMe}), 104.4(\mathrm{C} 3, \mathrm{C} 5), 104.7$ (C3', C5'), 118.5 (C3", C5"), 119.2 (C1, C1'), 120.2 (C1") , 124.8 (C2, C6), 129.1 (C2', C6'), 130.3 (C2"', C6"), 141.4 (C4), 153.9 $\left(\mathrm{C} 4^{\prime}\right), 155.9\left(\mathrm{C} 4^{\prime \prime}\right), 161.0(\mathrm{C}=\mathrm{N}), 162.4(\mathrm{C}=\mathrm{N})$. Anal. Calcd for $\mathrm{C}_{23} \mathrm{H}_{20} \mathrm{~N}_{2} \mathrm{O}_{5}: \mathrm{C}, 68.31 ; \mathrm{H}, 4.98 ; \mathrm{N}$ 6.93. Found: C, 67.92; H, 5.02; N 7.05.

2-(4-Methoxyphenyl)-5-(4-phenoxyphenyl)-1,3,4-oxadiazole (6c). White solid, Yield; 72\%, m.p.: 226-228 ${ }^{\circ} \mathrm{C}$. ${ }^{1} \mathrm{H}-\mathrm{NMR}\left(\mathrm{CDCl}_{3}\right)$ ): 3.87 (s, 3H, OMe), 7.00-7.02 (m, 2H, ArH), 7.10-7.20 (m, 4H, ArH), 7.17-7.20 (m, 1H, ArH), 7.37-7.40 (m, 2H, ArH), 8.03-8.07 (m, 4H, ArH). ${ }^{13} \mathrm{C}-\mathrm{NMR}\left(\mathrm{CDCl}_{3},\right)$ 8: $55.7(\mathrm{OMe}), 114.8$ (C3, C5), 116.7 (C3', C5'), 118.5 (C3", C5"), 118.7 (C1), 120.2 (C1'), 124.7 (C2, C6), 128.89 (C2', C6'), 128.93 (C2"), $130.27\left(\mathrm{C}^{\prime \prime}\right), 156.0(\mathrm{C} 4), 160.9\left(\mathrm{C} 4^{\prime}\right), 162.6\left(\mathrm{C} 4^{\prime \prime}\right), 1624.0(\mathrm{C}=\mathrm{N}), 166.6(\mathrm{C}=\mathrm{N})$. Anal. Calcd for $\mathrm{C}_{21} \mathrm{H}_{16} \mathrm{~N}_{2} \mathrm{O}_{3}$ : C, 73.24; $\mathrm{H}, 4.68 ; \mathrm{N}$ 8.13. Found: C, 73.62; H, 4.93; N 8.06.

2-(4-Nitrophenyl)-5-(4-phenoxyphenyl)-1,3,4-oxadiazole (6d). White solid, Yield; 77\%, m.p.: 226-228 ${ }^{\circ} \mathrm{C}$. ${ }^{1} \mathrm{H}-\mathrm{NMR}\left(\mathrm{CDCl}_{3}\right)$ 8: 7.09-7.13 (m, 4H, ArH), 7.19-7.24 (m, 1H, ArH), $7.42(\mathrm{dd}, J=15.8,8.0 \mathrm{~Hz}, 2 \mathrm{H}$, ArH), $7.42(\mathrm{dd}, J=16.0,8.0 \mathrm{~Hz}, 2 \mathrm{H}, \mathrm{ArH}), 8.28-8.45(\mathrm{~m}, 4 \mathrm{H}, \mathrm{ArH}) .{ }^{13} \mathrm{C}-\mathrm{NMR}\left(\mathrm{CDCl}_{3}\right)$ 8: $118.5(\mathrm{C} 3)$, 120.2 (C5), 120.4 (C3'), 124.7 (C5'), 124.8 (C3"), 125.0 (C5"), 128.0 (C1), 129.0 (C1'), 129.3 (C1"), 129.7 (C2), 130.3 (C6), 130.4 (C2', C6'), 149.7 (C2", C6") 155.6 (C4), 155.9 (C4'), 161.0 (C4"), 161.6 (C=N), 162.8 $(\mathrm{C}=\mathrm{N})$. Anal. Calcd for $\mathrm{C}_{20} \mathrm{H}_{13} \mathrm{~N}_{3} \mathrm{O}_{4}$ : $\mathrm{C}, 66.85 ; \mathrm{H}, 3.65 ; \mathrm{N}, 11.69$. Found: $\mathrm{C}, 66.73 ; \mathrm{H}, 3.39 ; \mathrm{N} 11.92$.

2-(4-Chlorophenyl)-5-(4-phenoxyphenyl)-1,3,4-oxadiazole (6e). White solid, Yield; 72\%, m.p.: 212-214 ${ }^{\circ} \mathrm{C}$. ${ }^{1} \mathrm{H}-\mathrm{NMR}\left(\mathrm{CDCl}_{3}\right)$ 8: 7.09-7.11 (m, 4H, ArH), 7.20-7.21 (m, 1H, ArH), 7.40-7.43 (m, 2H, ArH), 7.51-7.53 (m, 3H, ArH), 8.06-8.09 (m, 4H, ArH). ${ }^{13} \mathrm{C}-\mathrm{NMR}\left(\mathrm{CDCl}_{3}\right)$ 8: 118.5 (C3), 120.2 (C5), 120.3 (C3'), 122.4 (C5'), 122.7 (C3"), 124.7 (C5") 124.8 (C1), $128.4\left(\mathrm{C1}^{\prime}\right), 128.5$ (C1"), 129.0 (C2), 129.1 (C6), 129.7 (C2'), $129.8\left(\mathrm{C}^{\prime}\right), 130.28\left(\mathrm{C}^{\prime \prime}\right), 130.30\left(\mathrm{C}^{\prime \prime}\right), 138.2(\mathrm{C} 4), 138.4\left(\mathrm{C}^{\prime}\right), 155.8\left(\mathrm{C}^{\prime \prime}\right), 161.2(\mathrm{C}=\mathrm{N}), 164.2(\mathrm{C}=\mathrm{N})$. Anal. Calcd for $\mathrm{C}_{20} \mathrm{H}_{13} \mathrm{~N}_{3} \mathrm{O}_{4}$ : C, 68.87; H, 3.76; N, 8.03. Found: $\mathrm{C}, 68.63 ; \mathrm{H}, 3.40 ; \mathrm{N}$ 8.23.

2-(4-(Benzyloxy)phenyl)-5-(4-phenoxyphenyl)-1,3,4-oxadiazole (6f). White solid, Yield; 80\%, m.p.: 226-229 ${ }^{\circ} \mathrm{C} .{ }^{1} \mathrm{H}-\mathrm{NMR}\left(\mathrm{DMSO}-d_{6}\right)$ 8: $5.14\left(\mathrm{~s}, 2 \mathrm{H}, \mathrm{OCH}_{2}-\right), 7.03(\mathrm{~d}, J=8.3 \mathrm{~Hz}, 2 \mathrm{H}, \mathrm{ArH}), 7.06(\mathrm{~d}, J=8.1 \mathrm{~Hz}, 2 \mathrm{H}$, 
$\mathrm{ArH}), 7.12(\mathrm{t}, J=11.5 \mathrm{~Hz}, 2 \mathrm{H}, \mathrm{ArH}), 7.34-7.45(\mathrm{~m}, 8 \mathrm{H}, \mathrm{ArH}), 7.99(\mathrm{~d}, J=8.6 \mathrm{~Hz}, 2 \mathrm{H}, \mathrm{ArH}), 8.10(\mathrm{~d}$, $J=9.1 \mathrm{~Hz}, 2 \mathrm{H}, \mathrm{ArH}) .{ }^{13} \mathrm{C}-\mathrm{NMR}\left(\mathrm{DMSO}_{-} d_{6}\right) \delta: 70.9\left(\mathrm{OCH}_{2}-\right), 114.6(\mathrm{C} 3, \mathrm{C} 5) 114.8\left(\mathrm{C}^{\prime}, \mathrm{C}^{\prime}\right), 115.7\left(\mathrm{C} 3^{\prime \prime}\right)$,

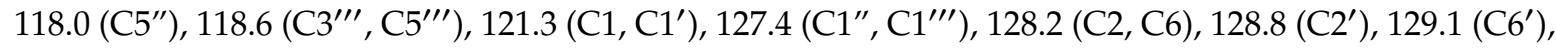
131.2 (C2", C6" $), 136.7\left(\mathrm{C} 2^{\prime \prime \prime}, \mathrm{C}^{\prime \prime \prime}{ }^{\prime \prime}\right), 153.5\left(\mathrm{C} 4, \mathrm{C} 4^{\prime}\right), 155.2\left(\mathrm{C} 4^{\prime \prime}\right), 157.3\left(\mathrm{C} 4^{\prime \prime \prime}\right), 165.7(\mathrm{C}=\mathrm{N}), 166.4(\mathrm{C}=\mathrm{N})$. Anal. Calcd for $\mathrm{C}_{27} \mathrm{H}_{20} \mathrm{~N}_{2} \mathrm{O}_{3}$ : C, 77.13; $\mathrm{H}, 4.79 ; \mathrm{N}$, 6.66. Found: $\mathrm{C}, 77.53 ; \mathrm{H}, 4.60 ; \mathrm{N}$ 6.24.

2-(Naphthalen-1-yl)-5-(4-phenoxyphenyl)-1,3,4-oxadiazole (6g). White solid, Yield; 70\%, m.p.: $173-175^{\circ} \mathrm{C}$. ${ }^{1} \mathrm{H}-\mathrm{NMR}\left(\mathrm{CDCl}_{3}\right)$ 8: 7.11-7.15 (m, 2H, ArH), 7.20-7.23 (m, 1H, ArH), 7.40-7.44 (m, 1H, ArH), 7.59-7.64 $(\mathrm{m}, 3 \mathrm{H}, \mathrm{ArH}), 7.69-7.75,(\mathrm{~m}, 1 \mathrm{H}, \mathrm{ArH}), 7.49-8.09(\mathrm{~m}, 3 \mathrm{H}, \mathrm{ArH}), 8.16(\mathrm{~d}, J=8.0 \mathrm{~Hz}, 1 \mathrm{H}, \operatorname{ArH}), 8.26(\mathrm{~d}$, $J=7 \mathrm{~Hz}, 1 \mathrm{H}, \mathrm{ArH}), 8.35(\mathrm{~d}, J=7 \mathrm{~Hz}, 1 \mathrm{H}, \mathrm{ArH}), 9.28(\mathrm{~d}, J=8.5 \mathrm{~Hz}, 1 \mathrm{H}, \mathrm{ArH}), 9.38(\mathrm{~d}, J=8.5 \mathrm{~Hz}, 1 \mathrm{H}$, ArH). ${ }^{13} \mathrm{C}-\mathrm{NMR}\left(\mathrm{CDCl}_{3}\right)$ 8: 118.6, 120.2, 120.8, 124.8, 125.1, 126.5, 127.0, 128.45, 128.50, 128.8, 128.9, 129.2, 130.3, 132.7, 132.9, 134.1, 134.2, 155.9, 161.1 (Ar-C), $164.1(\mathrm{C}=\mathrm{N}), 164.6(\mathrm{C}=\mathrm{N})$. Anal. Calcd for $\mathrm{C}_{24} \mathrm{H}_{16} \mathrm{~N}_{2} \mathrm{O}_{2}: \mathrm{C}, 79.11 ; \mathrm{H}, 4.43 ; \mathrm{N}, 7.69$. Found: $\mathrm{C}, 78.93 ; \mathrm{H}, 4.40 ; \mathrm{N} 8.01$.

2-(4-Phenoxyphenyl)-5-(pyridin-3-yl)-1,3,4-oxadiazole (6h). White solid, Yield; 70\%, m.p.: $143-144{ }^{\circ} \mathrm{C}$. ${ }^{1} \mathrm{H}-\mathrm{NMR}$ (DMSO- $\left.d_{6}\right) \delta: 7.03(\mathrm{~d}, J=7.5 \mathrm{~Hz}, 2 \mathrm{H}, \mathrm{ArH}), 7.06(\mathrm{~d}, J=8.2 \mathrm{~Hz}, 2 \mathrm{H}, \mathrm{ArH}), 7.19-7.22(\mathrm{~m}, 3 \mathrm{H}$, ArH), $7.39(\mathrm{t}, J=9.0 \mathrm{~Hz}, 2 \mathrm{H}, \mathrm{ArH}), 7.98(\mathrm{~d}, J=7.5 \mathrm{~Hz}, 2 \mathrm{H}, \mathrm{ArH}), 8.83(\mathrm{~d}, J=8.0 \mathrm{~Hz}, 2 \mathrm{H}, \mathrm{ArH}) ;{ }^{13} \mathrm{C}-\mathrm{NMR}$ (DMSO-d $\left.d_{6}\right) \delta: 114.9$ (C3, C5), 115.1 (C3', C5'), 118.3 (C5"-pyridyl), 120.5 (C1, C1'), 121.6 (C1"'-pyridyl), 123.5 (C2, C6), 127.3 (C2', C6'), 129.4 (C6"'-pyridyl), 131.2 (C4), 132.45 (C4'-pyridyl), 151.07 (C2=N pyridyl, $\mathrm{C} 4=\mathrm{N}$-pyridyl), $162.92(\mathrm{C}=\mathrm{N}), 165.62(\mathrm{C}=\mathrm{N})$. Anal. Calcd for $\mathrm{C}_{19} \mathrm{H}_{13} \mathrm{~N}_{3} \mathrm{O}_{2}: \mathrm{C}, 72.37 ; \mathrm{H}, 4.16$; N, 13.33. Found: C, 72.66; H, 4.39; N 13.65.

2-(4-Phenoxyphenyl)-5-(pyridin-4-yl)-1,3,4-oxadiazole (6i). White solid, Yield; 70\%, m.p.: $125-127^{\circ} \mathrm{C}$. ${ }^{1} \mathrm{H}-\mathrm{NMR}\left(\mathrm{CDCl}_{3}\right)$ ): 7.05-7.16 (m, 6H, ArH), 7.22-7.26 (m, 1H, ArH), 7.41-7.45 (m, 3H, ArH), 8.08-8.15 (m, 3H, ArH). ${ }^{13} \mathrm{C}-\mathrm{NMR}\left(\mathrm{CDCl}_{3}\right)$ 8: 117.5 (C3), 117.6 (C5), $117.9\left(\mathrm{C}^{\prime}\right), 118.2$ (C5'), 118.5 (C2, C6-pyridyl), 120.4 (C1), 120.5 (C1'), 120.7 (C1"'-pyridyl), 124.9 (C2), 129.5 (C6), 130.3 (C2'), 130.4 (C6'), 131.1 (C4), $132.6\left(\mathrm{C}^{\prime}\right), 133.1\left(\mathrm{C} 3=\mathrm{N}\right.$ pyridyl), $134.4\left(\mathrm{C} 5=\mathrm{N}\right.$ pyridyl), $162.7(2 \mathrm{C}=\mathrm{N})$. Anal. Calcd for $\mathrm{C}_{19} \mathrm{H}_{13} \mathrm{~N}_{3} \mathrm{O}_{2}$ : C, 72.37; H, 4.16; N, 13.33. Found: C, 72.54; H, 4.40; N 13.43 .

2-(Furan-2-yl)-5-(4-phenoxyphenyl)-1,3,4-oxadiazole (6j). White solid, Yield; 77\%, m.p.: $129-132{ }^{\circ} \mathrm{C}$. ${ }^{1} \mathrm{H}-\mathrm{NMR}\left(\mathrm{CDCl}_{3}\right) \delta: 6.65(\mathrm{~s}, 1 \mathrm{H}, \mathrm{ArH}), 7.11-7.13(\mathrm{~m}, 4 \mathrm{H}, \mathrm{ArH}), 7.23-7.25(\mathrm{~m}, 2 \mathrm{H}, \mathrm{ArH}), 7.44(\mathrm{t}, J=8.01$, 2H, ArH), $7.70(\mathrm{~s}, 1 \mathrm{H}, \mathrm{ArH}), 8.10(\mathrm{~d}, J=8.0 \mathrm{~Hz}, 2 \mathrm{H}, \mathrm{ArH}) .{ }^{13} \mathrm{C}-\mathrm{NMR}\left(\mathrm{CDCl}_{3}\right) \delta: 112.4(\mathrm{C} 3), 114.2(\mathrm{C} 5)$, $118.1\left(\mathrm{C}^{\prime}\right), 118.5\left(\mathrm{C}^{\prime}\right), 120.2$ (C1), $120.3\left(\mathrm{C1}^{\prime}\right), 124.8$ (C2-furfuryl), 129.0 (C5-furfuryl), 129.2 (C3, C4 furfuryl), $130.3(\mathrm{C} 2, \mathrm{C} 6), 139.8\left(\mathrm{C} 2^{\prime}\right), 145.9\left(\mathrm{C}^{\prime}\right), 155.8(\mathrm{C} 4), 157.5\left(\mathrm{C} 4^{\prime}\right), 161.2(\mathrm{C}=\mathrm{N}), 163.9(\mathrm{C}=\mathrm{N})$. Anal. Calcd for $\mathrm{C}_{18} \mathrm{H}_{12} \mathrm{~N}_{2} \mathrm{O}_{3}$ : C, 71.05; $\mathrm{H}, 4.67 ; \mathrm{N}, 9.21$. Found: C, 71.36; H, 4.39; N 9.55.

2-(4-Phenoxyphenyl)-5-(thiophen-2-yl)-1,3,4-oxadiazole (6k). White solid, Yield; 75\%, m.p.: 139-141 ${ }^{\circ} \mathrm{C}$. ${ }^{1} \mathrm{H}-\mathrm{NMR}\left(\mathrm{CDCl}_{3}\right)$ 8: 6.80-6.93 (m, 4H, ArH), 7.09-7.11 (m, 2H, ArH), 7.19-7.22 (m, 1H, ArH), 7.47-7.35 $(\mathrm{m}, 1 \mathrm{H}, \mathrm{ArH}), 8.07-8.09(\mathrm{~m}, 1 \mathrm{H}, \mathrm{ArH}), 7.81-7.83(\mathrm{~m}, 1 \mathrm{H}, \mathrm{ArH}) .{ }^{13} \mathrm{C}-\mathrm{NMR}\left(\mathrm{CDCl}_{3}\right)$ 8: $118.2(\mathrm{C} 3), 118.4$ (C5), 120.2 (C3'), 124.7 (C5'), 125.4 (C1), 128.3 (C1'), 128.9 (C2, C5-thienyl), 129.0 (C3, C4-thienyl), 129.8 (C2), $130.0(\mathrm{C} 6), 130.19\left(\mathrm{C}^{\prime}\right), 130.21\left(\mathrm{C}^{\prime}\right), 130.4(\mathrm{C} 4), 155.8\left(\mathrm{C} 4^{\prime}\right), 161.0(\mathrm{C}=\mathrm{N}), 163.9(\mathrm{C}=\mathrm{N})$. Anal. Calcd for $\mathrm{C}_{18} \mathrm{H}_{12} \mathrm{~N}_{2} \mathrm{O}_{2} \mathrm{~S}$ : C, 67.48; H, 3.78; N, 8.74. Found: C, 67.35; H, 3.36; N 9.02.

2,5-bis(4-Phenoxyphenyl)-1,3,4-oxadiazole (61). White solid, Yield; 85\%, m.p.: $232-234{ }^{\circ} \mathrm{C} .{ }^{1} \mathrm{H}-\mathrm{NMR}$ $\left(\mathrm{CDCl}_{3}\right)$ ): 6.95-7.12 (m, 9H, ArH), 7.17-7.24 (m, 2H, ArH), 7.32-7.40 (m, 4H, ArH), 8.04-8.406 (m, 3H, $\mathrm{ArH}) .{ }^{13} \mathrm{C}-\mathrm{NMR}\left(\mathrm{CDCl}_{3}\right) \delta: 118.1,118.5,118.6,118.9,119.3,119.5,120.2,123.9,124.7,129.0,129.5,129.8$, 130.0, 130.3, $155.9(\mathrm{Ar}-\mathrm{C}) 160.9(\mathrm{C}=\mathrm{N}), 164.2(\mathrm{C}=\mathrm{N})$. Anal. Calcd for $\mathrm{C}_{26} \mathrm{H}_{18} \mathrm{~N}_{2} \mathrm{O}_{3}: \mathrm{C}, 76.83 ; \mathrm{H}, 4.46 ; \mathrm{N}$, 6.89. Found: $\mathrm{C}, 77.01 ; \mathrm{H}, 4.60 ; \mathrm{N} 6.74$.

(E)-N'-Benzylidene-4-phenoxybenzohydrazide (8a). White solid, Yield; 89\%, m.p.: $163-165{ }^{\circ} \mathrm{C} .{ }^{1} \mathrm{H}-\mathrm{NMR}$ (DMSO- $\left.d_{6}\right) \delta:$ 7.08-7.12 (m, 4H, ArH), 7.12-7.24 (m, 1H, ArH), 7.44-7.47 (m, 5H, ArH), 7.73 (d, J = 6.9 $2 \mathrm{H}, \mathrm{ArH}), 7.96(\mathrm{~d}, J=8.2 \mathrm{~Hz}, 2 \mathrm{H}, \mathrm{ArH}), 8.46(\mathrm{~s}, 1 \mathrm{H}, \mathrm{CH}=\mathrm{N}), 11.82(\mathrm{~s}, 1 \mathrm{H}, \mathrm{NH}) .{ }^{13} \mathrm{C}-\mathrm{NMR}\left(\mathrm{DMSO}-d_{6}\right)$

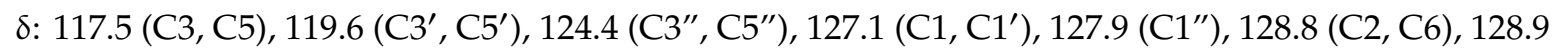


(C2', C6'), 130.0 (C2"), 130.3 (C6"), $134.4(\mathrm{C} 4), 147.5\left(\mathrm{C}^{\prime}\right), 155.5$ (C4"), $159.9(\mathrm{C}=\mathrm{O}), 162.4(\mathrm{C}=\mathrm{N})$. Anal. Calcd for $\mathrm{C}_{20} \mathrm{H}_{16} \mathrm{~N}_{2} \mathrm{O}_{2}$ : C, 75.93; H, 5.10; N, 8.86. Found: C, 75.54; H, 5.32; N 9.11.

(E)-4-Phenoxy-N'-(3,4,5-trimethoxybenzylidene)benzohydrazide (8b). White solid, Yield; 92\%, m.p.: 191-192 ${ }^{\circ} \mathrm{C} .{ }^{1} \mathrm{H}-\mathrm{NMR}$ (DMSO- $\left.d_{6}\right) \delta: 3.71$ (s, 3H, OMe), 3.84 (s, 6H, 2OMe), $7.03(\mathrm{~s}, 2 \mathrm{H}, \mathrm{ArH}), 7.10(\mathrm{~d}, J=7.9 \mathrm{~Hz}$, $4 \mathrm{H}, \mathrm{ArH}), 7.21-7.24(\mathrm{~m}, 1 \mathrm{H}, \mathrm{ArH}), 7.45(\mathrm{t}, J=7.9 \mathrm{~Hz}, 2 \mathrm{H}, \mathrm{ArH}), 7.95(\mathrm{~d}, J=8.0 \mathrm{~Hz}, 2 \mathrm{H}, \mathrm{ArH}), 8.39(\mathrm{~s}$, $1 \mathrm{H}, \mathrm{CH}=\mathrm{N}), 11.80(\mathrm{~s}, 1 \mathrm{H}, \mathrm{NH}) .{ }^{13} \mathrm{C}-\mathrm{NMR}$ (DMSO-d $\left.d_{6}\right) \delta: 55.9(\mathrm{OMe}), 602.1(2 \mathrm{OMe}), 104.3(\mathrm{C} 3, \mathrm{C} 5), 117.5$ $\left(\mathrm{C}^{\prime}{ }^{\prime}, \mathrm{C}^{\prime}\right), 119.6$ (C3", C5"), $124.4\left(\mathrm{C} 1, \mathrm{C1} 1^{\prime}\right), 128.0\left(\mathrm{C} 1^{\prime \prime}\right), 129.9$ (C2, C6), 130.3 (C2', C6'), 139.2 (C2", $\left.\mathrm{C6}^{\prime \prime}\right), 147.6(\mathrm{C} 4), 153.2\left(\mathrm{C}^{\prime}\right), 155.5\left(\mathrm{C}^{\prime \prime}\right), 159.9(\mathrm{C}=\mathrm{O}), 162.4(\mathrm{C}=\mathrm{N})$. Anal. Calcd for $\mathrm{C}_{23} \mathrm{H}_{22} \mathrm{~N}_{2} \mathrm{O}_{5}$ : $\mathrm{C}^{\prime \prime}$ 67.97; H, 5.46; N, 6.89. Found: C, 67.92; H, 5.32; N 7.02.

(E)-N'-(4-Methoxybenzylidene)-4-phenoxybenzohydrazide (8c). White solid, Yield; 92\%, m.p.: 233-235 ${ }^{\circ} \mathrm{C}$. ${ }^{1} \mathrm{H}-\mathrm{NMR}\left(\mathrm{DMSO}-d_{6}\right)$ 8: 3.81 (s, 3H, OMe), $7.02(\mathrm{~d}, J=8.4,2 \mathrm{H}, \mathrm{ArH}), 7.08-7.12(\mathrm{~m}, 4 \mathrm{H}, \mathrm{ArH}), 7.22(\mathrm{t}$, $J=7.5 \mathrm{~Hz}, 1 \mathrm{H}, \mathrm{ArH}), 7.45(\mathrm{t}, J=8.0 \mathrm{~Hz}, 2 \mathrm{H}, \mathrm{ArH}), 7.67(\mathrm{~d}, J=8.0 \mathrm{~Hz}, 2 \mathrm{H}, \mathrm{ArH}), 7.95(\mathrm{~d}, J=8.2 \mathrm{~Hz}, 2 \mathrm{H}$, $\mathrm{ArH}), 8.40(\mathrm{~s}, 1 \mathrm{H}, \mathrm{CH}=\mathrm{N}), 11.68(\mathrm{~s}, 1 \mathrm{H}, \mathrm{NH}) .{ }^{13} \mathrm{C}-\mathrm{NMR}$ (DMSO-d 6 ) $8: 55.3(\mathrm{OMe}), 114.3(\mathrm{C} 3, \mathrm{C} 5), 117.5$ $\left(\mathrm{C}^{\prime}, \mathrm{C}^{\prime}\right), 119.6\left(\mathrm{C}^{\prime \prime}, \mathrm{C} 5^{\prime \prime}\right), 124.4\left(\mathrm{C} 1, \mathrm{C1}^{\prime}\right), 126.9\left(\mathrm{C} 1^{\prime \prime}\right), 128.1(\mathrm{C} 2, \mathrm{C} 6), 128.6\left(\mathrm{C}^{\prime}, \mathrm{C} 6^{\prime}\right), 129.8\left(\mathrm{C} 2^{\prime \prime}\right)$, $130.3\left(\mathrm{C}^{\prime \prime}\right), 147.4(\mathrm{C} 4), 155.5\left(\mathrm{C}^{\prime}\right), 159.8\left(\mathrm{C}^{\prime \prime}\right), 160.8(\mathrm{C}=\mathrm{O}), 162.2(\mathrm{C}=\mathrm{N})$. Anal. Calcd for $\mathrm{C}_{21} \mathrm{H}_{18} \mathrm{~N}_{2} \mathrm{O}_{3}$ : C, 72.82; H, 5.24; N, 8.09. Found: C, 72.72; H, 5.53; N 8.06.

(E)-N'-(4-Chlorobenzylidene)-4-phenoxybenzohydrazide (8d). White solid, Yield; 92\%, m.p.: 184-186 ${ }^{\circ} \mathrm{C}$. ${ }^{1} \mathrm{H}-\mathrm{NMR}\left(\mathrm{DMSO}-d_{6}\right) \delta: 7.06(\mathrm{~d}, J=7 \mathrm{~Hz}, 2 \mathrm{H}, \mathrm{ArH}), 7.09(\mathrm{~d}, J=7.5 \mathrm{~Hz}, 2 \mathrm{H}, \operatorname{ArH}), 7.22(\mathrm{t}, J=11.5 \mathrm{~Hz}$, $2 \mathrm{H}, \mathrm{ArH}), 7.44(\mathrm{t}, J=12.0 \mathrm{~Hz}, 3 \mathrm{H}, \mathrm{ArH}), 7.99(\mathrm{~d}, J=8.4 \mathrm{~Hz}, 2 \mathrm{H}, \mathrm{ArH}), 8.10(\mathrm{~d}, J=9.2 \mathrm{~Hz}, 2 \mathrm{H}, \mathrm{ArH}), 8.77$ $(\mathrm{s}, 1 \mathrm{H}, \mathrm{CH}=\mathrm{N}), 11.86(\mathrm{~s}, 1 \mathrm{H}, \mathrm{NH}) .{ }^{13} \mathrm{C}-\mathrm{NMR}\left(\mathrm{DMSO}-d_{6}\right) \delta: 117.5(\mathrm{C} 3, \mathrm{C} 5), 119.6$ (C3', C5'), $124.4\left(\mathrm{C}^{\prime \prime}\right.$,

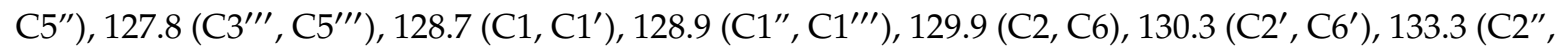
$\left.\mathrm{C}^{\prime \prime}\right), 134.4\left(\mathrm{C} 2^{\prime \prime \prime}, \mathrm{C} 6^{\prime \prime \prime}\right), 146.2\left(\mathrm{C} 4, \mathrm{C} 4^{\prime}\right), 155.4\left(\mathrm{C}^{\prime \prime}, \mathrm{C} 4^{\prime \prime \prime}\right), 160.0(\mathrm{C}=\mathrm{O}), 162.5(\mathrm{C}=\mathrm{N})$. Anal. Calcd for $\mathrm{C}_{20} \mathrm{H}_{15} \mathrm{ClN}_{2} \mathrm{O}_{2}$ : C, 68.48; H, 4.31; N, 7.99. Found: C, 68.37; H, 4.40; N 8.33.

(E)-N'-(4-Chlorobenzylidene)-4-phenoxybenzohydrazide (8e). White solid, Yield; $92 \%$, m.p.: $184-186{ }^{\circ} \mathrm{C}$. ${ }^{1} \mathrm{H}-\mathrm{NMR}$ (DMSO- $d_{6}$ ) $\delta: 7.09-7.12(\mathrm{~m}, 4 \mathrm{H}, \mathrm{ArH}), 7.23(\mathrm{t}, J=7.5 \mathrm{~Hz}, 2 \mathrm{H}, \mathrm{ArH}), 7.45(\mathrm{t}, J=7.5 \mathrm{~Hz}, 2 \mathrm{H}$, ArH), $7.97(\mathrm{~d}, J=8.0 \mathrm{~Hz}, 4 \mathrm{H}, \mathrm{ArH}), 8.29(\mathrm{~d}, J=8.4 \mathrm{~Hz}, 2 \mathrm{H}, \mathrm{ArH}), 8.54(\mathrm{~s}, 1 \mathrm{H}, \mathrm{CH}=\mathrm{N}), 12.10(\mathrm{~s}, 1 \mathrm{H}$,

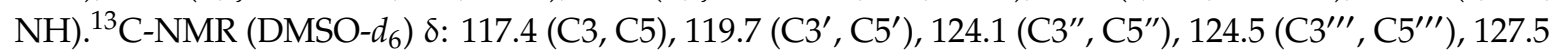

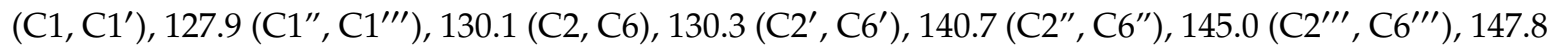
$\left(\mathrm{C} 4, \mathrm{C}^{\prime}\right), 148.8\left(\mathrm{C}^{\prime \prime}\right), 155.4\left(\mathrm{C}^{\prime \prime \prime}{ }^{\prime \prime}\right), 160.2(\mathrm{C}=\mathrm{O}), 162.6(\mathrm{C}=\mathrm{N})$. Anal. Calcd for $\mathrm{C}_{20} \mathrm{H}_{15} \mathrm{ClN}_{2} \mathrm{O}_{2}: \mathrm{C}, 68.48$; H, 4.31; N, 7.99. Found: C, 68.37; H, 4.40; N 8.33.

(E)-N'-[4-(Benzyloxy)benzylidene]-4-phenoxybenzohydrazide (8f). White solid, Yield; 93\%, m.p.: 228-231 ${ }^{\circ} \mathrm{C} .{ }^{1} \mathrm{H}-\mathrm{NMR}$ (DMSO- $\left.d_{6}\right)$ 8: 5.17 (s, 2H, ArH), 7.08-7.12 (m, 6H, ArH), 7.21-7.24 (m, 1H, ArH), 7.33-7.48 $(\mathrm{m}, 7 \mathrm{H}, \mathrm{ArH}), 7.67(\mathrm{~d}, J=8.7 \mathrm{~Hz}, 2 \mathrm{H}, \mathrm{ArH}), 7.94(\mathrm{~d}, J=8.6 \mathrm{~Hz}, 2 \mathrm{H}, \mathrm{ArH}), 8.39(\mathrm{~s}, 1 \mathrm{H}, \mathrm{CH}=\mathrm{N}), 11.68(\mathrm{~s}$, $1 \mathrm{H}, \mathrm{NH}) .{ }^{13} \mathrm{C}-\mathrm{NMR}\left(\mathrm{DMSO}-d_{6}\right) \delta: 69.4\left(\mathrm{OCH}_{2}\right), 115.2(\mathrm{C} 3), 117.5(\mathrm{C} 5), 119.6\left(\mathrm{C}^{\prime}, \mathrm{C}^{\prime}\right), 124.4\left(\mathrm{C}^{\prime \prime}\right)$, 127.1 (C5"), 127.8 (C1), 127.9 (C1'), 128.1 (C1"), 128.5 (C2), 128.6 (C6), 129.8 (C2', C6'), 130.3 (C2", C6"), $136.8(\mathrm{C} 4), 147.4\left(\mathrm{C}^{\prime}\right), 155.5\left(\mathrm{C}^{\prime \prime}\right), 159.9(\mathrm{C}=\mathrm{O}), 162.2(\mathrm{C}=\mathrm{N})$. Anal. Calcd for $\mathrm{C}_{27} \mathrm{H}_{22} \mathrm{~N}_{2} \mathrm{O}_{3}: \mathrm{C}, 76.76 ; \mathrm{H}$, $5.25 ; \mathrm{N}, 6.63$. Found: $\mathrm{C}, 76.56 ; \mathrm{H}, 5.60 ; \mathrm{N} 6.34$.

(E)-N'-(Naphthalen-1-ylmethylene)-4-phenoxybenzohydrazide (8g). White solid, Yield; 88\%, m.p.: 193-195 ${ }^{\circ} \mathrm{C} .{ }^{1} \mathrm{H}-\mathrm{NMR}\left(\mathrm{DMSO}-d_{6}\right) \delta:$ 7.12-7.14 (m, 4H, ArH), $7.24(\mathrm{t}, J=7.9 \mathrm{~Hz}, 2 \mathrm{H}, \mathrm{ArH}), 7.45-7.48(\mathrm{~m}, 2 \mathrm{H}$, ArH), 7.60-7.62, (m, 2H, ArH), 7.63-7.69, (m, 1H, ArH), 7.93-7.95 (m, 1H, ArH), 8.01-8.04 (m, 4H, ArH), 8.87-8.89 (m, 1H, ArH), $9.11(\mathrm{~s}, 1 \mathrm{H}, \mathrm{CH}=\mathrm{N}), 11.90(\mathrm{~s}, 1 \mathrm{H}, \mathrm{NH}) .{ }^{13} \mathrm{C}-\mathrm{NMR}$ (DMSO- $\left.d_{6}\right) \delta: 117.5,119.6$, 124.2, 124.4, 125.6, 126.3, 127.3, 127.7, 127.9, 128.8, 129.6, 129.9, 130.2, 130.3, 130.5, 133.5, 147.4, 155.5 (Ar-C), $160.0(\mathrm{C}=\mathrm{O}), 162.3(\mathrm{C}=\mathrm{N})$. Anal. Calcd for $\mathrm{C}_{24} \mathrm{H}_{18} \mathrm{~N}_{2} \mathrm{O}_{2}$ : C, 78.67; H, 4.95; N, 7.65. Found: $\mathrm{C}$, 78.92; H, 4.60; N 8.04.

(E)-4-Phenoxy-N'-(pyridin-3-ylmethylene)benzohydrazide (8h). White solid, Yield; 90\%, m.p.: $149-151^{\circ} \mathrm{C}$. ${ }^{1} \mathrm{H}-\mathrm{NMR}$ (DMSO- $d_{6}$ ) $\delta:$ 7.09-7.14 (m, 4H, ArH), 7.22-7.25 (m, 1H, ArH), 7.44-7.50 (m, 3H, ArH), 7.97 $(\mathrm{d}, J=8.4 \mathrm{~Hz}, 2 \mathrm{H}, \mathrm{ArH}), 8.14(\mathrm{~d}, J=7.0 \mathrm{~Hz}, 1 \mathrm{H}, \mathrm{ArH}), 8.51(\mathrm{~s}, 1 \mathrm{H}, \mathrm{CH}=\mathrm{N}), 8.62(\mathrm{~d}, J=8.3 \mathrm{~Hz}, 1 \mathrm{H}, \mathrm{ArH})$, 
$8.86(\mathrm{~d}, J=8.3 \mathrm{~Hz}, 1 \mathrm{H}, \mathrm{ArH}), 11.98(\mathrm{~s}, 1 \mathrm{H}, \mathrm{NH}) .{ }^{13} \mathrm{C}-\mathrm{NMR}\left(\mathrm{DMSO}-d_{6}\right) \delta: 117.5(\mathrm{C} 3, \mathrm{C} 5), 119.6\left(\mathrm{C}^{\prime}, \mathrm{C}^{\prime}\right)$, 124.0 (C5-pyridyl), 124.5 (C1), 127.7 (C1'), 130.0 (C1-pyridyl), 130.3 (C2, C6), 133.4 (C2', C6'), 144.8 (C6-pyridyl), $148.7\left(\mathrm{C} 4, \mathrm{C} 4^{\prime}\right), 150.7(\mathrm{C} 2=\mathrm{N}$ pyridyl), $155.4(\mathrm{C} 4=\mathrm{N}$ pyridyl), $160.0(\mathrm{C}=\mathrm{O}), 162.5(\mathrm{C}=\mathrm{N})$. Anal. Calcd for $\mathrm{C}_{19} \mathrm{H}_{15} \mathrm{~N}_{3} \mathrm{O}_{2}$ : C, 71.91; $\mathrm{H}, 4.76 ; \mathrm{N}, 13.24$. Found: $\mathrm{C}, 71.46 ; \mathrm{H}, 4.54 ; \mathrm{N} 13.55$.

(E)-4-Phenoxy-N'-(pyridin-4-ylmethylene)benzohydrazide (8i). White solid, Yield; $92 \%$, m.p.: $175-177^{\circ} \mathrm{C}$. ${ }^{1} \mathrm{H}-\mathrm{NMR}$ (DMSO- $\left.d_{6}\right) \delta: 7.10-7.13(\mathrm{~m}, 4 \mathrm{H}, \mathrm{ArH}), 7.24(\mathrm{t}, J=8.2 \mathrm{~Hz}, 1 \mathrm{H}, \mathrm{ArH}), 7.46(\mathrm{t}, J=8.2 \mathrm{~Hz}, 2 \mathrm{H}$, $\mathrm{ArH}), 7.67(\mathrm{~s}, 2 \mathrm{H}, \mathrm{ArH}), 7.97(\mathrm{~d}, J=7.9 \mathrm{~Hz}, 2 \mathrm{H}, \mathrm{ArH}), 8.44(\mathrm{~s}, 1 \mathrm{H}, \mathrm{CH}=\mathrm{N}), 8.66(\mathrm{~s}, 2 \mathrm{H}, \mathrm{ArH}), 12.08(\mathrm{~s}$, $1 \mathrm{H}, \mathrm{NH}) .{ }^{13} \mathrm{C}-\mathrm{NMR}$ (DMSO- $\left.d_{6}\right) \delta$ : 117.5 (C3, C5), 119.7 (C3', C5'), 121.0 (C5-pyridyl), 124.5 (C1, C1'), 127.5 (C1-pyridyl), 130.1 (C2, C6), 130.3 (C2', C6'), 141.5 (C6-pyridyl), 145.0 (C4, C4'), 150.3 (C3=N pyridyl), $155.4\left(\mathrm{C} 4=\mathrm{N}\right.$ pyridyl), $160.2(\mathrm{C}=\mathrm{O}), 162.6(\mathrm{C}=\mathrm{N})$. Anal. Calcd for $\mathrm{C}_{19} \mathrm{H}_{15} \mathrm{~N}_{3} \mathrm{O}_{2}: \mathrm{C}, 71.91 ; \mathrm{H}$, 4.76; N, 13.24. Found: C, 71.64; H, 4.60; N 13.42.

(E)-N'-(Furan-2-ylmethylene)-4-phenoxybenzohydrazide (8j). White solid, Yield; 89\%, m.p.: 192-194 ${ }^{\circ} \mathrm{C}$. ${ }^{1} \mathrm{H}-\mathrm{NMR}$ (DMSO- $\left.d_{6}\right): \delta 6.64(\mathrm{~s}, 1 \mathrm{H}, \mathrm{ArH}), 6.92(\mathrm{~s}, 1 \mathrm{H}, \mathrm{ArH}), 7.08-7.12(\mathrm{~m}, 4 \mathrm{H}, \mathrm{ArH}), 7.21-7.24(\mathrm{~m}$, $1 \mathrm{H}, \mathrm{ArH}), 7.45(\mathrm{t}, J=7.9 \mathrm{~Hz}, 2 \mathrm{H}, \mathrm{ArH}), 7.85(\mathrm{~s}, 1 \mathrm{H}, \mathrm{ArH}), 7.93(\mathrm{~d}, J=7.9 \mathrm{~Hz}, 2 \mathrm{H}, \mathrm{ArH}), 8.34(\mathrm{~s}, 1 \mathrm{H}$, $\mathrm{CH}=\mathrm{N}), 11.75(\mathrm{~s}, 1 \mathrm{H}, \mathrm{NH}) .{ }^{13} \mathrm{C}-\mathrm{NMR}\left(\mathrm{DMSO}^{-} d_{6}\right) \delta: 112.2(\mathrm{C} 3), 133.4(\mathrm{C} 5), 117.5\left(\mathrm{C}^{\prime}, \mathrm{C}^{\prime}\right), 119.6$ (C3, C4-furfuryl), 124.4 (C1), 127.9 (C1'), 129.9 (C2, C6), 130.3 (C2', C6'), 137.3 (C2-furfuryl), 145.1 (C5-furfuryl), $149.5(\mathrm{C} 4), 155.5\left(\mathrm{C} 4^{\prime}\right), 159.9(\mathrm{C}=\mathrm{O}), 162.3(\mathrm{C}=\mathrm{N})$. Anal. Calcd for $\mathrm{C}_{18} \mathrm{H}_{14} \mathrm{~N}_{2} \mathrm{O}_{3}$ : C, 70.58 ; $\mathrm{H}, 4.61 ; \mathrm{N}, 9.15$. Found: $\mathrm{C}, 70.37 ; \mathrm{H}, 4.32 ; \mathrm{N} 9.50$.

(E)-4-Phenoxy-N'-(thiophen-2-ylmethylene)benzohydrazide (8k). White solid, Yield; 92\%, m.p.: $182-183{ }^{\circ} \mathrm{C}$. ${ }^{1} \mathrm{H}-\mathrm{NMR}$ (DMSO- $\left.d_{6}\right) \delta:$ 7.08-7.24 (m, 6H, ArH), 7.44-7.47 (m, 3H, ArH), $7.67(\mathrm{~d}, J=7.5 \mathrm{~Hz}, 1 \mathrm{H}, \mathrm{ArH})$, $7.93(\mathrm{~d}, J=7.0 \mathrm{~Hz}, 2 \mathrm{H}, \mathrm{ArH}), 8.66(\mathrm{~s}, 1 \mathrm{H}, \mathrm{CH}=\mathrm{N}), 11.76(\mathrm{~s}, 1 \mathrm{H}, \mathrm{NH}) .{ }^{13} \mathrm{C}-\mathrm{NMR}$ (DMSO- $\left.d_{6}\right) \delta: 117.5(\mathrm{C} 3$, C5), 119.6 (C3', C5'), 124.4 (C3, C4 thienyl), 127.8 (C1) $128.8\left(\mathrm{C1}^{\prime}\right), 128.8$ (C2, C6), $129.8\left(\mathrm{C2}^{\prime}\right), 130.3$ (C6') $130.8(\mathrm{C} 4), 139.2\left(\mathrm{C} 4^{\prime}\right), 142.7$ (C2-thienyl), $155.5(\mathrm{C} 4-$ thienyl), $159.9(\mathrm{C}=\mathrm{O}), 162.2(\mathrm{C}=\mathrm{N})$. Anal. Calcd for $\mathrm{C}_{18} \mathrm{H}_{14} \mathrm{~N}_{2} \mathrm{O}_{2} \mathrm{~S}$ : C, 67.06; $\mathrm{H}, 4.38 ; \mathrm{N}$, 8.69. Found: $\mathrm{C}, 67.36 ; \mathrm{H}, 3.39 ; \mathrm{N} 9.04$.

\subsection{Biology}

\subsubsection{In Vitro Anti-Tubercular Activity}

The MICs of Compounds 3, 4, 6a-1, and $\mathbf{8 a}-\mathbf{k}$ for M. tuberculosis H37Rv were determined by the microplate Alamar blue assay (MABA) (Table 1) [31,32]. Triclosan and INH were used as reference standards. M. tuberculosis H37Rv (ATCC 27294; American Type Culture Collection, Rockville, MD, USA) was grown to late log phase in Middlebrook 7H9 broth. Twofold dilutions of tested drugs were prepared in 7H9 broth in a volume of $100 \mu \mathrm{L}$ in 96-well, clear-bottom microplates at a final testing concentration of $0.2,0.4,0.8,1.6,3.12,6.25,12.5,25,50$ and $100 \mu \mathrm{g} / \mathrm{mL}$. $100 \mu \mathrm{L}$ of M. tuberculosis H37Rv (containing $2 \times 10^{4} \mathrm{CFU}$ ) was added, yielding a final testing volume of $200 \mu \mathrm{L}$. In each row, a drug-free (bacteria-only) control was added.

The plates were incubated at $37^{\circ} \mathrm{C}$; on the seventh day of incubation, $12.5 \mu \mathrm{L}$ of $20 \%$ Tween 80 and $20 \mu \mathrm{L}$ of Alamar blue were added to all wells. After incubation at $37^{\circ} \mathrm{C}$ for 16 to $24 \mathrm{~h}$, the fluorescence of the wells was read at an excitation of $530 \mathrm{~nm}$ and emission of $590 \mathrm{~nm}$. The MIC was defined as the lowest concentration affecting a reduction in fluorescence of $\geq 90 \%$ relative to the mean of replicate bacteria-only controls.

Among the tested compounds, the results displayed that most of them i.e., $\mathbf{3}, \mathbf{4}, \mathbf{6 h}, \mathbf{6 i}, \mathbf{6 1}, \mathbf{8 g}$, and $8 \mathbf{k}$ showed promising anti-tubercular activity (MIC, $0.61-1.69 \mu \mathrm{g} / \mathrm{mL}$ ) in comparison to triclosan (MIC, $10 \mu \mathrm{g} / \mathrm{mL}$ ) and INH (MIC, $0.2 \mu \mathrm{g} / \mathrm{mL}$ ).

\subsubsection{In Vitro InhA Enzyme Inhibition}

InhA enzyme assay was carried out utilizing a Cary100 Bio spectrophotometer (Agilent Technologies, Santa Clara, California, USA) at $25^{\circ} \mathrm{C}$, through monitoring the oxidation of NADH to NAD ${ }^{+}$at $340 \mathrm{~nm}$. Reactions were initiated via the addition of dodecenoyl-CoA $(50 \mathrm{~mm})$ substrate to mixtures containing 
InhA (5 nm), NADH (100 mm), and inhibitor $(1-10,000 \mathrm{~nm})$. The $\mathrm{IC}_{50}$ values were determined from a dose-response plot of enzyme fractional activity versus the concentration of inhibitor.

\subsubsection{Cytotoxic Activity}

The cytotoxicity test was carried out using Vero cell line (African green monkey kidney epithelial cells), obtained from American Type Culture Collection (ATCC). The cells were cultured according to ATCC recommendations as it was described earlier [41,42]. To evaluate cytotoxicity, $3 \times 10^{4}$ Vero cells were seeded in the wells of 96-multiwell plates in $100 \mu \mathrm{L}$ of the complete culture medium. After $24 \mathrm{~h}$ incubation at $37{ }^{\circ} \mathrm{C}$ when the cells were well attached and achieved $90 \%$ confluence, the culture medium was replaced with $100 \mu \mathrm{L}$ of derivative solutions, which were tested at the concentration range of $0.1-200 \mu \mathrm{g} / \mathrm{mL}$. The stock solutions of the synthesized derivatives were prepared in DMSO and the tested concentrations were prepared by nine serial 2-fold dilutions. Vero cells were exposed to the compounds and rifampicin for $24 \mathrm{~h}$ and then cell viability was assessed by MTT assay (Sigma-Aldrich Chemicals, St. Louis, MO, USA). The $\mathrm{IC}_{50}$ value, which is defined as the concentration of the derivative causing reduction in cell viability by 50\%, was calculated using GraphPad Prism 5, version 5.03 software. Selectivity/safety index (SI) for tested agents was determined using the formula: $\mathrm{SI}=\mathrm{IC}_{50} / \mathrm{MIC}$.

\subsubsection{Docking Methodology}

Discovery Studio 2.5 software (Accelrys Inc., San Diego, CA, USA) was used for docking analysis. Fully automated docking tool using "Dock ligands (CDOCKER)" protocol running on Intel (R) Core (TM) i32370 CPU @ 2.4 GHz, RAM Memory 2 GB under the Windows 7.0 system. The crystal structures of InhA reductase enzyme (PDB code: 3FNH) were downloaded from the protein data bank [36-38]. See the Supplementary Materials.

\section{Conclusions}

A set of diphenyl ether hydrazides and their cyclized oxadiazole analogs were designed and synthesized as triclosan analogs. The targeted compounds have been identified using spectroscopic data and elemental microanalysis. The antitubercular activity of the un-substituted oxadiazole 3 and the hydrazide 4 experienced the highest activity than triclosan as a reference drug. Arylated oxadiazoles $6 \mathbf{i}$ and $\mathbf{6}$ and arylidenes $\mathbf{8 g}$ and $\mathbf{8 k}$ showed potent antitubercular activity against Mycobacterium tuberculosis H37Rv strains. The InhA reductase enzymatic study supports the experimental data. We believe there is more than one mode of mechanisms are involved in the antimycobacterial property of the synthesized diphenyl ether derivatives. The obtained results from the in silico pharmacokinetic properties calculation showed that most of the target compounds exhibited drug-like characters. Taken together, a docking study has boosted that the higher activity of compounds 3,4 , and $8 \mathrm{~g}$ could be further investigated as potential lead antimycobacterial candidates.

Supplementary Materials: The following are available online: general experimental data and NMR spectra.

Author Contributions: Conceptualization, T.S.I. and S.S.P.; Methodology, A.M.M.A.-M., E.S.T., A.M.M., A.N.K., R.M.B. and I.A.S.; Software, M.F.A.M.; Validation, T.S.I. and S.S.P.; Formal analysis, E.S., A.M.M., A.N.K., R.M.B. and I.A.S.; Investigation, T.S.I., E.S.T., M.A.A., and S.S.P.; Resources, H.Z.A., N.A.A.; Data curation, T.S.I. and S.S.P.; Writing — original draft preparation, T.S.I., E.S.T., M.F.A.M. and S.S.P.; Writing—review and editing, T.S.I., E.S.T. and S.S.P.; Visualization, T.S.I. and S.S.P.; Supervision, T.S.I. and S.S.P.; Project administration, H.Z.A., N.A.A.; Funding acquisition, H.Z.A., N.A.A. All authors have read and agreed to the published version of the manuscript.

Funding: This research was funded by King Abdulaziz University, Jeddah, under grant No. (RG-009-166-38).

Acknowledgments: The authors gratefully acknowledge DSR, King Abdulaziz University, Jeddah, for technical and financial support No. (RG-009-166-38).

Conflicts of Interest: The authors declare no conflict of interest. 


\section{References}

1. World Health Organization. Global Tuberculosis Report. 2015. Available online: www.who.int/tb/ publications/global_report/gtbr15_main_text.pdf (accessed on 27 January 2020).

2. World Health Organization. Global Tuberculosis Report. 2019. Available online: https://www.who.int/newsroom/events/detail/2019/03/24/default-calendar/world-tb-day-2019 (accessed on 27 January 2020).

3. Matteelli, A.; Roggi, A.; Carvalho, A.C. Extensively drug-resistant tuberculosis: Epidemiology and management. Clin. Epidemiol. 2014, 6, 111-118. [CrossRef] [PubMed]

4. Chollet, A.; Maveyraud, L.; Lherbet, C.; Bernardes-Genisson, V. An overview on crystal structures of InhA protein: Apo-form, in complex with its natural ligands and inhibitors, Eur. J. Med. Chem. 2018, 146, 318-343. [CrossRef]

5. Ende, C.W.A.; Knudson, S.E.; Liu, N.; Childs, J.; Sullivan, T.J.; Boyne, M.; Xu, H.; Gegina, Y.; Knudson, D.L.; Johnson, F.; et al. Synthesis and in vitro antimycobacterial activity of B-ring modified diaryl ether InhA inhibitors. Bioorg. Med. Chem. Lett. 2008, 18, 3029-3033. [CrossRef] [PubMed]

6. Perozzo, R.; Kuo, M.; Sidhu, A.B.S.; Valiyaveetti, J.T.; Bittman, R.; Jacobs, W.R., Jr.; Fidock, D.A.; Sacchettini, J.C.J. Structural Elucidation of the Specificity of the Antibacterial Agent Triclosan for Malarial Enoyl Acyl Carrier Protein Reductase. Biol. Chem. 2002, 277, 13106-13114. [CrossRef] [PubMed]

7. Sharma, S.; Ramya, T.N.C.; Surolia, A.; Surolia, N. Triclosan as a Systemic Antibacterial Agent in a Mouse Model of Acute Bacterial Challenge. Antimicrob. Agents Chemother. 2003, 47, 3859-3866. [CrossRef]

8. Chhibber, M.; Kumar, G.; Parasuraman, P.; Ramya, T.N.; Surolia, N.; Surolia, A. Novel diphenyl ethers: Design, docking studies, synthesis and inhibition of enoyl ACP reductase of Plasmodium falciparum and Escherichia coli. Bioorg. Med. Chem. 2006, 14, 8086-8098. [CrossRef]

9. Mishra, S.; Karmodiya, K.; Parasuraman, P.; Surolia, A.; Surolia, N. Design, synthesis, and application of novel triclosan prodrugs as potential antimalarial and antibacterial agents. Bioorg. Med. Chem. Lett. 2008, 16, 5536-5546. [CrossRef]

10. Butler, M.S.; Cooper, M.A.J. Antibiotics in the clinical pipeline in 2011. J. Antibiot. 2011, 64, 413-425. [CrossRef] [PubMed]

11. Tonge, P.J.; Kisker, C.; Slayden, R.A. Development of Modern InhA Inhibitors to Combat Drug Resistant Strains of Mycobacterium tuberculosis. Curr. Top. Med. Chem. 2007, 7, 489-498. [CrossRef]

12. Kini, S.G.; Bhat, A.R.; Bryant, B.; Williamson, J.S.; Dayan, F.E. Synthesis, antitubercular activity and docking study of novel cyclic azole substituted diphenyl ether derivatives. Eur. J. Med. Chem. 2009, 44, 492-500. [CrossRef]

13. Kini, S.G.; Bhat, A.R.; Pan, Z.; Dayan, F.E. Synthesis and antitubercular activity of heterocycle substituted diphenyl ether derivatives. J. Enzyme Inhib. Med. Chem. 2010, 25, 730-736. [CrossRef] [PubMed]

14. Sivaraman, S.; Sullivan, T.J.; Johnson, F.; Novichenok, P.; Cui, G.; Simmerling, C.; Tonge, P.J. Inhibition of the Bacterial Enoyl Reductase FabI by Triclosan: A Structure-Reactivity Analysis of FabI Inhibition by Triclosan Analogues. J. Med. Chem. 2004, 47, 509-518. [CrossRef] [PubMed]

15. Heath, R.J.; Yu, Y.T.; Shapiro, M.A.; Olson, E.; Rock, C.O. Broad spectrum antimicrobial biocides target the FabI component of fatty acid synthesis. J. Biol. Chem. 1998, 273, 30316-30320. [CrossRef] [PubMed]

16. Yang, Y.H.; Wang, Z.L.; Yang, J.Z.; Yang, T.; Pi, W.Y.; Ang, W.; Lin, Y.N.; Liu, Y.Y.; Li, Z.C.; Luo, Y.F.; et al. Design, synthesis and evaluation of novel molecules with a diphenyl ether nucleus as potential antitubercular agents. Bioorg. Med. Chem. Lett. 2012, 22, 954-957. [CrossRef] [PubMed]

17. Navarrete-Vazquez, G.; Molina-Salinas, G.M.; Duarte-Fajardo, Z.V.; Vargas-Villarreal, J.; Estrada-Soto, S.; Gonzalez-Salazar, F.; Hernandez-Nunez, E.; Said-Fernandez, S. Synthesis and antimycobacterial activity of 4-(5-substituted-1,3,4-oxadiazol-2-yl)pyridines. Bioorg. Med. Chem. 2007, 15, 5502-5508. [CrossRef] [PubMed]

18. Khan, R.H.; Rastogi, R.C. Synthesis of some 2-[(5-aryl-1,3,4-oxadiazol-2-yl)amino]-1,3-heterazoles as potential pesticides. J. Agric. Food Chem. 1990, 38, 1068-1071. [CrossRef]

19. Narayana, B.; Raj, K.K.V.; Ashalatha, B.V.; Kumari, N.S. Synthesis of Some New 2-(6-Methoxy-2-Naphthyl) -5-Aryl-1,3,4-Oxadiazoles as Possible Non-steroidal Anti-inflammatory and Analgesic Agents. Arch. Pharm. Chem. Life Sci. 2005, 338, 373-377. [CrossRef]

20. Rasras, A.J.M.; Al-Tel, T.H.; Al-Aboudi, A.F.; Al-Qawasmeh, R.A. Synthesis and antimicrobial activity of cholic acid hydrazone analogues. Eur. J. Med. Chem. 2010, 45, 2307-2313. [CrossRef] 
21. Mohareb, R.M.; Fleita, D.H.; Sakka, O.K. Novel Synthesis of Hydrazide-Hydrazone Derivatives and Their Utilization in the Synthesis of Coumarin, Pyridine, Thiazole and Thiophene Derivatives with Antitumor Activity. Molecules 2011, 16, 16-27. [CrossRef]

22. Cascioferro, S.; Attanzio, A.; Di Sarno, V.; Musella, S.; Tesoriere, L.; Cirrincione, G.; Diana, P.; Parrino, B. New 1,2,4-Oxadiazole Nortopsentin Derivatives with Cytotoxic Activity. Mar. Drugs 2019, 17, 35. [CrossRef]

23. Rollas, S.; Küçükgüzel, S.G. Biological Activities of Hydrazone Derivatives. Molecules 2007, 12, $1910-1939$. [CrossRef]

24. Asif, M. Pharmacologically potentials of hydrazonone containing compounds: A promising scaffold. Int. J. Adv. Chem. 2014, 2, 85-103. [CrossRef]

25. Oliveira, P.F.M.; Guidetti, B.; Chamayou, A.; André-Barrès, C.; Madacki, J.; Korduláková, J.; Mori, G.; Orena, B.S.; Chiarelli, L.R.; Pasca, M.R.; et al. Mechanochemical Synthesis and Biological Evaluation of Novel Isoniazid Derivatives with Potent Antitubercular Activity. Molecules 2017, 22, 1457. [CrossRef]

26. Freundlich, J.S.; Wang, F.; Vilcheze, C.; Gulten, G.; Langley, R.; Schiehser, G.A.; Jacobus, D.R.; Jacobs, W.R.; Sacchettini, J.C. Triclosan derivatives: Towards potent inhibitors of drug-sensitive and drug-resistant Mycobacterium tuberculosis. Chem. Med. Chem. 2009, 4, 241-248. [CrossRef] [PubMed]

27. Ibrahim, H.R.; Tatsumoto, S.; Ono, H.; Van Immerseel, F.; Raspoet, R.; Miyata, T. A novel antibiotic-delivery system by using ovotransferrin as targeting molecule. Eur. J. Pharm. Sci. 2015, 66, 59-69. [CrossRef] [PubMed]

28. Haga, N.; Takayanagi, H. Mechanisms of the Photochemical Rearrangement of Diphenyl Ethers. J. Org. Chem. 1996, 61, 735-745. [CrossRef]

29. He, L.; Zhang, L.; Liu, X.; Li, X.; Zheng, M.; Li, H.; Yu, K.; Chen, K.; Shen, X.; Jiang, H.; et al. Discovering Potent Inhibitors Against the $\beta$-Hydroxyacyl-Acyl Carrier Protein Dehydratase (FabZ) of Helicobacter pylori: Structure-Based Design, Synthesis, Bioassay, and Crystal Structure Determination. J. Med. Chem. 2009, 52, 2465-2481. [CrossRef]

30. Ibrahim, T.S.; Al-Mahmoudy, A.M.; Elagawany, M.; Ibrahim, M.A.; Panda, S.S. Synthesis and Antiviral Bioassay of New Diphenyl Ether-based Compounds. Chem. Biol. Drug Des. 2016, 88, 511-518. [CrossRef]

31. Collins, L.; Franzblau, S.G. Microplate alamar blue assay versus BACTEC 460 system for high-throughput screening of compounds against Mycobacterium tuberculosis and Mycobacterium avium. Antimicrob. Agents Chemother. 1997, 41, 1004-1009. [CrossRef]

32. Franzblau, S.G.; Witzig, R.S.; McLaughlin, J.C.; Torres, P.; Madico, G.; Hernandez, A.; Degnan, M.T.; Cook, M.B.; Quenzer, V.K.; Ferguson, R.M.; et al. Rapid, low-technology MIC determination with clinical Mycobacterium tuberculosis isolates by using the microplate Alamar Blue assay. J. Clin. Microbiol. 1998, 36, 362-366. [CrossRef]

33. Umaa, K.; Sudha Rani, S.; Sangeetha, G.; Maida Engels, S.E. Prediction of physiochemical parameters for anti-tubercular potentials of Mannich and Schiff bases. J. Chem. Pharm. Res. 2013, 5, 52-59.

34. Lakshminarayana, S.B.; Huat, T.B.; Ho, P.C.; Manjunatha, U.H.; Dartois, V.; Dick, T.; Rao, S.P.S. Comprehensive physicochemical, pharmacokinetic and activity profiling of anti-TB agents. J. Antimicrob. Chemother. 2015, 70, 857-867. [CrossRef] [PubMed]

35. Wiener, H. Structural determination of Paraffin boiling point. J. Am. Chem. Soc. 1947, 69, 17-20. [CrossRef]

36. Stec, J.; Vilchèze, C.; Lun, S.; Perryman, A.L.; Wang, X.; Freundlich, J.S.; Bishai, W.; Jacobs, W.R., Jr.; Kozikowski, A.P. Biological evaluation of potent triclosan-derived inhibitors of the enoyl-acyl carrier protein reductase InhA in drug-sensitive and drug-resistant strains of Mycobacterium tuberculosis. Chem. Med. Chem. 2014, 9, 2528-2537. [CrossRef] [PubMed]

37. Mohamed, M.F.A.; Youssif, B.G.M.; Shaykoon, M.S.A.; Abdelrahman, M.H.; Elsadek, B.E.M.; Aboraia, A.S.; Abuo-Rahma, G.E.A. Utilization of tetrahydrobenzo[4,5]thieno[2,3-d]pyrimidinone as a cap moiety in design of novel histone deacetylase inhibitors. Bioorg. Chem. 2019, 91, 103127. [CrossRef] [PubMed]

38. Youssif, B.G.M.; Mohamed, M.F.A.; Al-Sanea, M.M.; Moustafa, A.H.; Abdelhamid, A.A.; Gomaa, H.A.M. Novel aryl carboximidamides and 3-aryl-1,2,4-oxadiazoles analogues of naproxen as dual selective COX-2 / LOX inhibitors: Design, synthesis and Docking studies. Bioorg. Chem. 2019, 85, 577-584. [CrossRef] [PubMed]

39. Liu, Y.; Li, G.; Yang, L. Cu(I)-catalyzed Synthesis of Diaryl Ether Using a Simple Hydrazone as Promoter. Chin. J. Chem. 2009, 27, 423-427. [CrossRef]

40. Bar-Yuan, H.; Kun-Ming, Y.; Yun, C. Synthesis of electroluminescent copoly(aryl ether)s containing alternate 1,4-distyrylbenzene derivatives and aromatic 1,3,4-oxadiazole or 3,3"-terphenyldicarbonitrile segments. J. Polym. Sci. Pol. Chem. 2005, 43, 5009-5022. 
41. Sieniawska, E.; Sawicki, R.; Swatko-Ossor, M.; Napiorkowska, A.; Przekora, A.; Ginalska, G.; Augustynowicz-Kopec, E. The effect of combining natural terpenes and antituberculous agents against reference and clinical Mycobacterium tuberculosis strains. Molecules 2018, 23, 176. [CrossRef]

42. Trotsko, N.; Golus, J.; Kazimierczak, P.; Paneth, A.; Przekora, A.; Ginalska, G.; Wujec, M. Synthesis and antimycobacterial activity of thiazolidine-2,4-dione based derivatives with halogenbenzohydrazones and pyridinecarbohydrazones substituents. Eur. J. Med. Chem. 2020, 189, 112045. [CrossRef]

Sample Availability: Samples of the compounds $\mathbf{6 a}-\mathbf{1}$ and $\mathbf{8 a}-\mathbf{k}$ are available from the authors.

(C) 2020 by the authors. Licensee MDPI, Basel, Switzerland. This article is an open access article distributed under the terms and conditions of the Creative Commons Attribution (CC BY) license (http://creativecommons.org/licenses/by/4.0/). 